\title{
Human adipose-derived mesenchymal progenitor cells plus microfracture and hyaluronic acid for cartilage repair: a Phase Ila trial
}

\author{
Zhiguang Qiao ${ }^{\ddagger 1,2}$, Jiaxin Tang ${ }^{\ddagger 1,3}$, Bing Yue ${ }^{4}$, Jinwu Wang ${ }^{1}$, Jun Zhang ${ }^{1}$, Liang Xuan ${ }^{1}$, \\ Chengxiang Dai ${ }^{5}$, Suke $\mathrm{Li}^{5}$, Meng $\mathrm{Li}^{5}$, Cuili Xu${ }^{5}$, Kerong Dai*,4 \& You Wang**,1,2 \\ ${ }^{1}$ Shanghai Key Laboratory of Orthopaedic Implants, Department of Orthopaedic Surgery, Shanghai Ninth People's Hospital, \\ Shanghai Jiao Tong University School of Medicine, Shanghai 200011, China \\ ${ }^{2}$ Medical 3D Printing Innovation Research Center, Shanghai Jiao Tong University School of Medicine, Shanghai 200125, China \\ ${ }^{3}$ Department of Bone \& Joint Surgery, Shanghai Fourth People's Hospital Affiliated to Tongji University School of Medicine, \\ Shanghai 200081, China \\ ${ }^{4}$ Department of Bone \& Joint Surgery, Renji Hospital, Shanghai Jiao Tong University School of Medicine, Shanghai 200020, China \\ ${ }^{5}$ Cellular Biomedicine Group, Shanghai 201210, China \\ *Author for correspondence: drwangyou@163.com \\ **Author for correspondence: krdai@163.com \\ $\ddagger$ Authors contributed equally
}

\begin{abstract}
Aim: This study aimed to preliminarily evaluate the safety and efficacy of human adipose-derived mesenchymal progenitor cells (haMPCs) in combination with microfracture and hyaluronic acid (HA) for treating cartilage defects. Materials \& methods: A total of 30 patients with medial femoro-tibial condylar cartilage defects were randomized into three groups: arthroscopic microfracture group and normal saline injection, arthroscopic microfracture and intra-articular injection of $\mathrm{HA}$, or arthroscopic microfracture in combination with intra-articular injection of HA and haMPCs. Results \& conclusions: The data demonstrated that intra-articular injection of haMPCs plus microfracture and HA is a safe procedure to improve joint function in patients with knee cartilage defects. These findings provide an impetus for future research on this treatment. ClinicalTrials.gov Identifier: NCT02855073
\end{abstract}

First draft submitted: 31 May 2019; Accepted for publication: 13 January 2020; Published online: 11 February 2020

Keywords: Artz $\bullet$ cartilage defect $\bullet$ cartilage regeneration $\bullet$ human adipose-derived mesenchymal progenitor cells $\bullet$ microfracture $\bullet$ Re-Join ${ }^{\circledR}$ 


\section{Graphical abstract:}

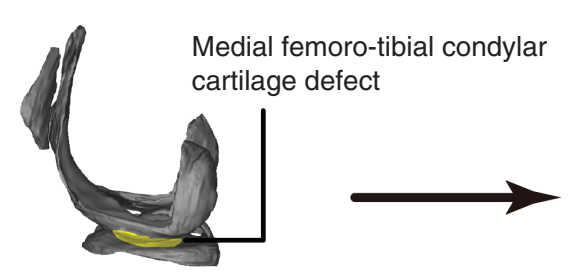

\section{Microfracture +} HA \& Re-Join ${ }^{\circledast}$
injection (MSR)
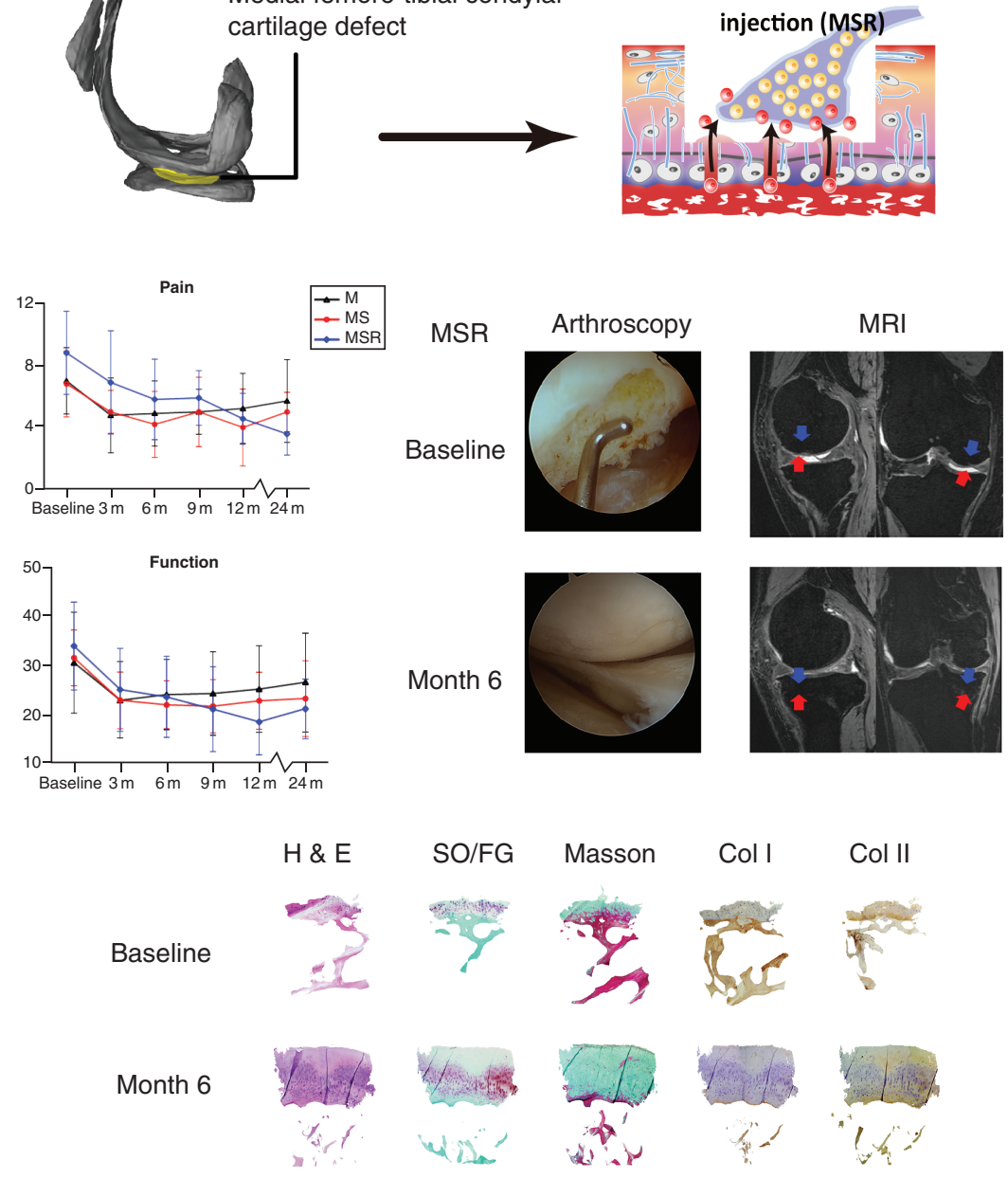

Col I Col II

$3 \mathrm{D}$ reconstruction
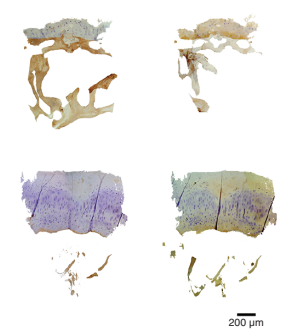

$20 \overline{\mathrm{mm}}$
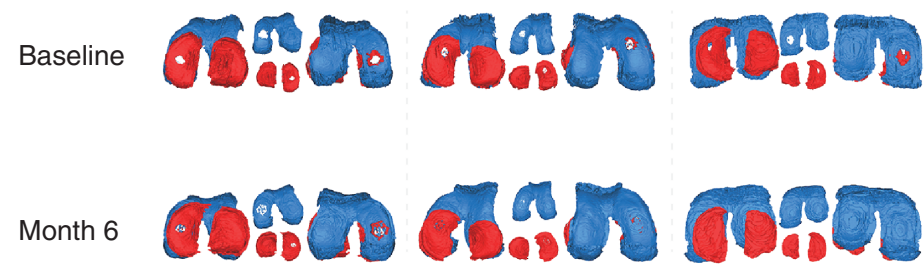

Knee osteoarthritis (OA) is a degenerative joint disease, leading to irreversible damage of the cartilage, osteophyte formation, bone reorganization, synovial inflammation and loss of joint function [1,2]. Current treatments focus on relieving symptoms and improving joint function [3,4]. Total joint replacement is proven to be the most effective surgical intervention; however, it is an aggressive procedure and should be reserved for patients whose symptoms have not responded to other treatments [5]. Other treatment strategies include but are not limited to (arthroscopic) microfracture, intra-articular injection of hyaluronic acid (HA) and intra-articular injection of stem cells.

Although clinical efficacy of microfracture [6-10], intra-articular injection of HA [11-20] and stem cells [21-26] have been reported by multiple published studies, these treatments were used mainly in younger patients with low grade OA, making it difficult to evaluate the treatment efficacy in Kellgren Lawrence (KL) grade 3 and KL grade 4 OA. 
In addition, many studies have cast doubt on the value of these treatments, indicating that microfracture $[27,28]$ and HA [29] provide no additional benefit over physiotherapy and placebo. Although a knee arthroplasty is predictable and reasonable for high-grade OA, it may not be ideal for all patients. After total knee arthroplasty (TKA), lowimpact activities, instead of moderate- or high-impact activities, are recommended [30,31]. Only 20\% patients return to high impact sports [32]. Active patients younger than 60 years with symptomatic moderate to severe OA are frequently looking for alternative treatment options, even if only a temporizing measure [33].

In previous studies, various stem cell sources have been used to treat OA because of their chondrogenic and anti-inflammatory properties [34-36]. Among them, adipose-derived stem cells (ADSCs) are easy to collect for clinical application with high isolation yields and are not affected by the patients' age, sex and physiological status [37]. Like stem cells, progenitor cells also tend to differentiate into a specific type of cell and replicate themselves to replace or repair damaged tissues or organs. Results in previous studies suggested that intra-articular injection of human adipose-derived mesenchymal progenitor cells (haMPCs) in animal models promoted regeneration of articular cartilage [38,39].

To date, few studies have used haMPCs in treating cartilage defects in moderate to severely arthritic knees. This study, therefore, aimed to preliminarily evaluate the safety and efficacy of haMPCs in KL grade 3 patients with knee cartilage defects. We conducted a Phase IIa proof-of-concept clinical trial in KL grade 3 patients aged 50-75 with knee cartilage defects to preliminarily evaluate and compare the clinical effects of arthroscopic microfracture, intraarticular injection of sodium hyaluronic acid (Artz, Seikagaku Corporation, Japan), and intra-articular injection of haMPCs (Re-Join ${ }^{\circledR}$, Cellular Biomedicine Group, Inc., USA). We assumed that haMPCs in combination with microfracture and HA will provide clinical improvement and promote cartilage regeneration for patients with cartilage defects within arthritic knees.

\section{Patients \& methods}

A randomized, single-blind, parallel group, Phase IIa proof-of-concept clinical trial to preliminarily evaluate and compare the clinical effects of arthroscopic microfracture, sodium hyaluronic acid and Re-Join ${ }^{\circledR}$ (autologous adipose-derived mesenchymal progenitor cells and sodium hyaluronic acid) injection for the patients with cartilage defects within arthritic knees was conducted from July 2016 to December 2018 in two hospitals: Shanghai Ninth People's Hospital Affiliated to Shanghai Jiao Tong University School of Medicine and Renji Hospital Affiliated to Shanghai Jiao Tong University School of Medicine. The study protocol was approved by the local ethics committees of both institutions (No.22, 2014; Scientific Research Projects Approval Determination of Independent Ethics Committee of Shanghai Ninth People's Hospital affiliated to Shanghai Jiao Tong University School of Medicine and Scientific Research Projects Approval Determination of Independent Ethics Committee of Renji Hospital Affiliated to Shanghai Jiao Tong University School of Medicine) and registered at clinicaltrials.gov with identifier NCT02855073 (date of registration, July 8, 2016). The methods were carried out in accordance with the relevant guidelines and regulations. Informed consent was obtained according to the Helsinki Declaration.

\section{Patient selection \& enrollment}

Patients with a clinical diagnosis of knee $\mathrm{OA}$ with medial femoro-tibial condylar or trochlear-patellar cartilage defect were assessed for eligibility. Based on the defect area, enrolled patients were separated into two groups. Informed Consent was obtained from all patients before participation in this trial.

After signing the Informed Consent Form, each patient was randomized into one of the three treatment groups: The M group, treated with arthroscopic microfracture and intra-articular injection of normal saline (NS); the MS group, treated with arthroscopic microfracture and intra-articular injection of sodium hyaluronic acid; or the MSR group, treated with arthroscopic microfracture in combination with intra-articular injection of sodium hyaluronic acid and Re-Join. A study flowchart detailing this information is shown in Figure 1. Analgesics were not permitted except for the rescue medications, acetaminophen, at a dose of $4000 \mathrm{mg}$ or less per day.

\section{Inclusion criteria}

Eligible patients were between 50 and 75 years of age with clinical diagnosis of knee OA cartilage defects and radiographic changes of grade 3 according to Kellgren-Lawrence criteria in the targeted knee [40] and had an average pain intensity of grade 6 or more on a 10-point visual analog scale (VAS) for at least 4 months. To be included in the study, the patient must have either medial femoro-tibial condylar defect or trochlear-patellar defect 
A Medial femoro-tibial condylar cartilage defect

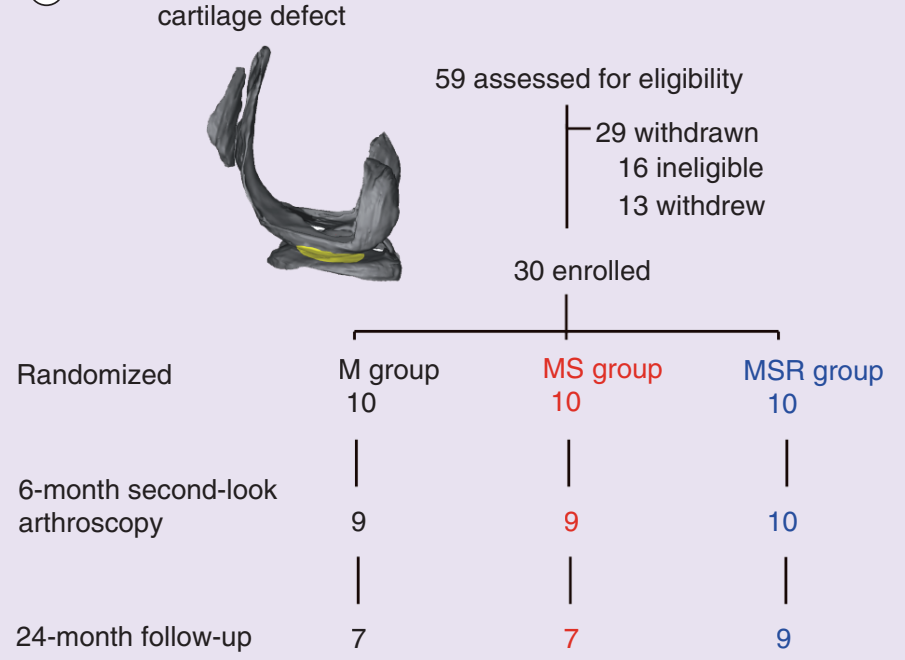

Trochlear-patellar cartilage defect

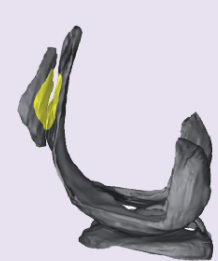

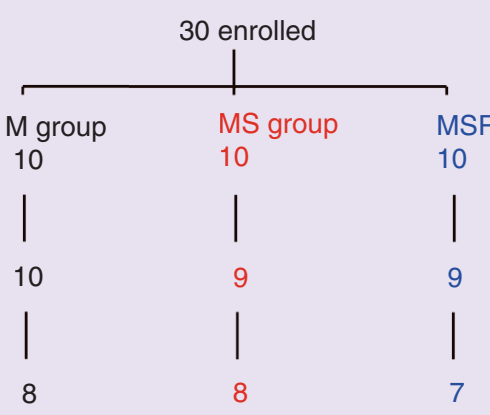

(B)
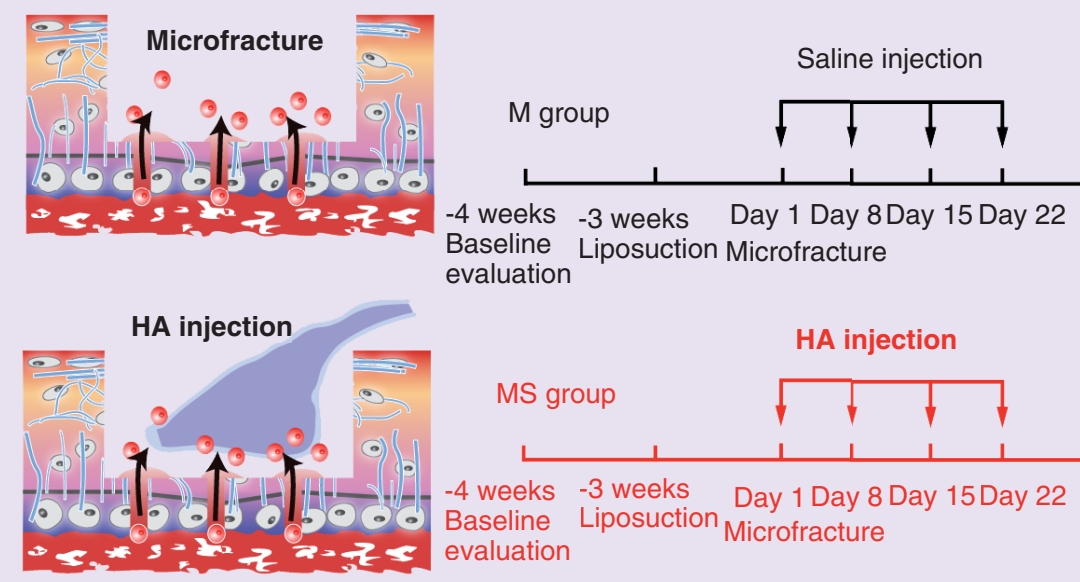

Follow up:

$3,6,9,12,24$ months

Second-look arthroscopy \& MRI: 6 months
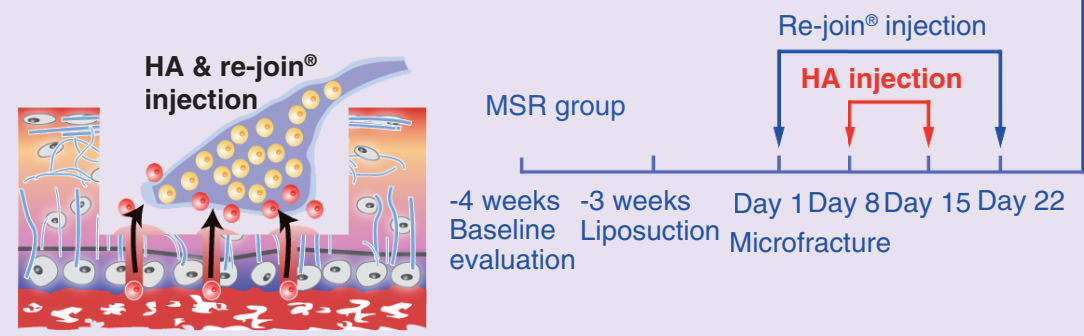

Figure 1. Study flow diagram. (A) Flowchart of the clinical trial. (B) Diagrams for each treatment (left) and detailed time points for treatment and follow-ups (right).

HA: Hyaluronic acid; M: Arthroscopic microfracture and normal saline injections; MRI: Magnetic resonance imaging; MS: Arthroscopic microfracture and sodium hyaluronic acid injections; MSR: Arthroscopic microfracture, sodium hyaluronic acid, and Re-Join ${ }^{\circledR}$ injections.

and the defect area must have been $2-6 \mathrm{~cm}^{2}$ measured by MRI, and the course of knee OA must have been no longer than 10 years.

\section{Exclusion criteria}

Patients were excluded if they met at least one of the following criteria: had both medial femoro-tibial condylar and trochlear-patellar cartilage defect; pregnant women or lactating mothers; previous arthroscopic treatment for knee OA; intra-articular HA or corticosteroid injection in the preceding 3 months; previous total meniscectomy, 
meniscal allograft, bucket-handle tears and complex tears; secondary arthritis (related to rheumatoid arthritis, spondyloarthritis, previous major knee trauma, inflammatory or postinfectious arthritis, and crystal arthropathies); positive human immunodeficiency (HIV), hepatitis B (HBV) or hepatitis C (HCV) at screening indicative of current of pass infection; major malalignment which can affect this study such as uncontrollable cardiovascular diseases, renal diseases, liver diseases, endocrine diseases, cancer or diabetes; participated in other clinical trials within 3 months prior to this study; or patients deemed to be not suitable for the study by the investigator for other reasons.

\section{Treatment randomization}

Eligible participants who met criteria were randomly assigned using central randomization software (SAS, version 9.4) 1:1:1 to the M group (receiving arthroscopic microfracture treatment and NS injections), MS group (receiving arthroscopic microfracture treatment and sodium hyaluronic acid injections) and MSR group (receiving arthroscopic microfracture treatment, sodium hyaluronic acid and Re-Join injections). Using computer-generated random numbers, Tang J generated the random allocation sequence and assigned participants to interventions. Whereas physicians were aware of the allocated arm, patients and outcome assessors were kept blinded to the allocation.

\section{About Re-Join therapy \& sodium hyaluronic acid}

Re-Join is a product composed of in vitro expanded autologous MPCs derived from adipose tissue of patients combined with cell suspension solution (see Supplementary Method 1 for detailed information). Our preliminary experiment with dose escalation for the adipose MSCs has shown that the dosage of $5 \times 10^{7}$ haMSCs exhibited the highest improvement [41]. Sodium HA used in this study was Artz (Seikagaku Corporation, Japan; see Supplementary Method 3 for detailed information).

\section{Treatment}

All procedures were performed in the supine position under general anesthesia. A single orthopedic surgeon performed all procedures. The procedures were arthroscopic, and the principles of the technique introduced by Steadman et al. [10] were used. Articular cartilage lesions were measured with a calibrated arthroscopic probe. To obtain stable and healthy cartilage edges, debridement of all damaged and unstable cartilage was performed. Microfractures were performed at all defect areas (medial femoro-tibial condyles or trochlea and patella) with an arthroscopic awl. The holes were 3-4 mm apart from each other and deep enough to show the 'fat-pearls' from the subchondral bone. For patients with medial femoro-tibial condylar cartilage defect, all the defects in medial femoro-tibial compartment were microfractured. For trochlear-patellar cartilage defects, all the defects in trochlear-patellar compartment were microfractured. Cartilage defects in other areas were not treated. In patients who gave consent, a 2-mm-punch biopsy specimen was obtained from the center of the cartilage defect of the medial femoral condyle. The day on which the arthroscopic surgery was performed was marked as day 1 . For the M group, subjects received NS injections on days 1, 8, 15 and 22. For the MS group, subjects received sodium hyaluronic acid injections on days 1, 8, 15 and 22. For the MSR group, subjects received Re-Join injections on days 1 and 22 and sodium hyaluronic acid injections on days 8 and 15. A study flowchart detailing this information is shown in Figure 1B. Postoperative rehabilitation program started from day 1. All patients were educated using crutches and avoiding weight bearing for 4 weeks. A stepwise increase in load bearing was introduced after that.

After completing the 24-month observation, all patients can receive follow-up treatment with Re-Join.

\section{Second-look arthroscopy}

Second-look arthroscopy 6 months post-treatment was conducted by two surgeons at our hospital. Cartilage defects were measured with a calibrated arthroscopic probe, and the healing status was visually inspected and scored as per the validated International Cartilage Repair Society (ICRS) [42] macroscopic score. In case of multiple cartilage defects, the size of each defect was summed up. The observation was confirmed only once a consensus was reached by both two surgeons. A 2-mm punch biopsy specimen was obtained from the adjacent area to the first biopsy site in patients who gave consent. 


\section{Outcome measures}

The primary end points were the safety and the Western Ontario and McMaster Universities Arthritis Index (WOMAC) [43] total index (0-96 scale) at month 12 after treatment. The safety was systematically assessed with vital signs, physical examination, laboratory tests and adverse events at each visit throughout the study.

Secondary efficacy end points included: WOMAC physical function (0-68 scale), pain (0-20 scale) and stiffness (0-8 scale) subscales and quality of life measured by the short-form 36 (SF-36) questionnaire [44] (time frame: 0 day, 3 months, 6 months, 9 months, 12 months and 24 months). The size of the cartilage defect was measured using MRI by two independent blinded radiologist specialize in musculoskeletal reads [45]. For calculating size of the cartilage defect, the maximum diameter of the cartilage defect in each compartment was measured in the sagittal (anteroposterior) and coronal (mediolateral) images measured using imaging processing software (Mimics 15.0 Materialise Corporation, Leuven, Belgium). Size of the cartilage defect was calculated by multiplying the two diameters. In case of multiple cartilage defects, the size of each defect was summed up. This also applied to follow-ups. Changes in the cartilage volume and the defected area were measured using Mimics 15.0 (Materialise Corporation, Leuven, Belgium) by a single observer (ZQ) blinded to other results (time frame: 0 day and 6 months; see Supplementary Method 2). The defect size and healing status of degenerated cartilage was measured with a calibrated arthroscopic probe and the ICRS macroscopic score [46,47] during the second-look arthroscopy (time frame: 0 day and 6 months). For histological assessment, biopsy specimens were subject to hematoxylin-eosin (H\&E) staining, safranin O/fast green (SOFG) staining, Masson's trichrome staining and immunohistochemistry for type I and II collagen. Hematoxylin staining was used as a counter-stain for immunohistochemistry. Thickness of regenerated cartilage was measured, and specimens were evaluated with ICRS II (time frame: 0 day and 6 months) [48]. Inflammation parameter was proved difficult to score in analyzed biopsy specimens in previous study thus not scored in our study.

\section{Statistical analysis}

Based on the result from previous studies, for each treatment group, 8 participants were required to achieve $85 \%$ power and an $\alpha$ level of 0.05 (one-way analysis of variance). Thus, 24 participants were required. Considering a compliance rate of $80 \%, 30$ participants were planned to be randomly assigned to allow for dropouts.

Outcome measures were analyzed based on the intention-to-treat population. All values are expressed as number $(\%)$ of patients or mean \pm standard deviation. The significance of differences between groups was assessed using one-way analysis of variance (one-way ANOVA) for parametric variables, or Kruskal-Wallis ANOVA test and a Dunn-Bonferroni test for post hoc comparisons for nonparametric variables. Changes from baseline to treatment visits were assessed with paired-samples t-test for parametric variables, or Mann-Whitney U test for nonparametric variables within the group. A value of $\mathrm{p}<0.05$ was considered statistically significant. All analyses were performed using IBM SPSS Statistics version 19.0 for Windows (IBM Corporation, NY, USA).

\section{Results}

\section{Characteristics of patients}

A total of 59 patients with medial femoro-tibial condylar cartilage defects were assessed for eligibility and 30 patients were enrolled. For patients with trochlear-patellar defect, 67 patients were screened and 30 were enrolled. Eligible participants were randomly assigned to treatment (for patients with medial femoro-tibial condylar defect, 10 in $\mathrm{M}$, 10 in MS and 10 in MSR each group; for patients with trochlear-patellar defect, 10 in each group; Figure 1A). All treatment groups had similar baseline characteristics (Tables $1 \& 2$ ).

For patients who had medial femoro-tibial condylar cartilage defects, 28 patients underwent second-look arthroscopy 6 months post-treatment and 23 patients completed the 24-month follow-up. For patients who had trochlear-patellar cartilage defects, 28 underwent second-look arthroscopy after 6 months. 23 patients completed the 24-month follow-up. However, due to lack of consent for biopsy on second-look arthroscopy (only four patients gave consent), patients with trochlear-patellar cartilage defects were excluded from following analysis.

\section{Adverse effects}

AEs are summarized in Table 3. No serious AEs were observed in this study. No local or systemic safety concerns were indicated through vital signs, physical examinations or laboratory tests during each follow-up. Several minor AEs were potentially related to the procedures: slight joint swelling and pain in treated knees were reported by five patients, in which three patients it was resolved with intermittent acetaminophen and in two patients the swelling 
Table 1. Baseline characteristics of patients with medial femoro-tibial cartilage defect.

\begin{tabular}{|c|c|c|c|c|}
\hline Characteristic & $M(n=10)$ & MS $(n=10)$ & MSR $(n=10)$ & p-value \\
\hline Age (year) & $62.3 \pm 5.27$ & $59.7 \pm 7.12$ & $62.0 \pm 8.33$ & 0.667 \\
\hline Women & $7(70)$ & $5(50)$ & $7(70)$ & 0.574 \\
\hline BMI $\left(\mathrm{kg} / \mathrm{m}^{2}\right)$ & $25.4 \pm 3.16$ & $26.4 \pm 2.14$ & $26.0 \pm 2.86$ & 0.705 \\
\hline \multicolumn{5}{|l|}{ Kellgren-Lawrence system } \\
\hline - Grade III & $10(100)$ & $10(100)$ & $10(100)$ & 1.000 \\
\hline - Global knee pain VAS $(0-10 \mathrm{~cm})$ & $7.57 \pm 1.42$ & $7.44 \pm 1.00$ & $6.85 \pm 1.04$ & 0.355 \\
\hline \multicolumn{5}{|l|}{ WOMAC } \\
\hline - Total index (0-96 scale) & $40.2 \pm 12.9$ & $40.9 \pm 6.26$ & $45.8 \pm 10.2$ & 0.419 \\
\hline - Function subscale (0-68 scale) & $30.3 \pm 10.4$ & $31.3 \pm 5.81$ & $33.7 \pm 9.08$ & 0.669 \\
\hline - Pain subscale (0-20 scale) & $7.00 \pm 2.16$ & $6.80 \pm 2.15$ & $8.80 \pm 2.70$ & 0.131 \\
\hline - Stiffness subscale ( $0-8$ scale) & $2.90 \pm 2.08$ & $2.80 \pm 1.99$ & $3.30 \pm 1.34$ & 0.813 \\
\hline \multicolumn{5}{|l|}{ SF-36 } \\
\hline - Physical component & $48.8 \pm 5.47$ & $51.1 \pm 4.85$ & $48.4 \pm 5.84$ & 0.500 \\
\hline - Mental component & $53.0 \pm 7.93$ & $49.9 \pm 8.75$ & $54.0 \pm 5.94$ & 0.466 \\
\hline \multicolumn{5}{|c|}{$\begin{array}{l}\text { Data are number (\%) of patients or mean } \pm \text { standard deviation values. Differences in parameters between groups were analyzed using one-way ANOVA for continuous variables, } \\
\text { or Kruskal-Wallis ANOVA test and a Dunn-Bonferroni test for post hoc comparisons for categorical variables. } \\
\text { BMI: Body mass index; M: Microfracture and saline; MS: Microfracture and sodium hyaluronic acid; MSR: Microfracture, sodium hyaluronic acid and Re-Join }{ }^{\circledR} \text {; SF-36: Short-form } \\
36 \text { (quality of life); VAS: Visual analog scale; WOMAC: Western Ontario and McMaster University Osteoarthritis Index. }\end{array}$} \\
\hline
\end{tabular}

\section{Table 2. Baseline characteristics of patients with trochlear-patellar cartilage defect.}

\begin{tabular}{|c|c|c|c|c|}
\hline Characteristic & $M(n=10)$ & MS $(n=10)$ & MSR $(n=10)$ & p-value \\
\hline Age, year & $62.0 \pm 6.39$ & $64.1 \pm 7.62$ & $60.2 \pm 9.21$ & 0.545 \\
\hline Women & $7(70)$ & $7(70)$ & $6(60)$ & 0.865 \\
\hline $\mathrm{BMI}, \mathrm{kg} / \mathrm{m}^{2}$ & $26.2 \pm 3.80$ & $24.7 \pm 3.97$ & $25.3 \pm 4.13$ & 0.694 \\
\hline \multicolumn{5}{|l|}{ Kellgren-Lawrence system } \\
\hline - Grade III & $10(100)$ & $10(100)$ & $10(100)$ & 1.000 \\
\hline - Global knee pain VAS $(0-10 \mathrm{~cm})$ & $7.57 \pm 1.42$ & $7.44 \pm 0.906$ & $6.72 \pm 1.71$ & 0.350 \\
\hline \multicolumn{5}{|l|}{ WOMAC } \\
\hline - Total index (0-96 scale) & $42.8 \pm 9.02$ & $37.5 \pm 8.24$ & $38.7 \pm 7.26$ & 0.332 \\
\hline - Function subscale (0-68 scale) & $32.7 \pm 8.62$ & $28.1 \pm 8.18$ & $28.8 \pm 8.01$ & 0.419 \\
\hline - Pain subscale (0-20 scale) & $7.80 \pm 3.29$ & $6.70 \pm 2.67$ & $6.00 \pm 2.58$ & 0.380 \\
\hline - Stiffness subscale (0-8 scale) & $2.30 \pm 1.77$ & $2.70 \pm 1.34$ & $3.90 \pm 1.91$ & 0.107 \\
\hline \multicolumn{5}{|l|}{ SF-36 } \\
\hline - Physical component & $47.4 \pm 4.05$ & $51.5 \pm 7.18$ & $49.4 \pm 9.83$ & 0.484 \\
\hline - Mental component & $51.3 \pm 5.33$ & $54.4 \pm 7.44$ & $53.0 \pm 7.96$ & 0.617 \\
\hline \multicolumn{5}{|c|}{$\begin{array}{l}\text { Data are number (\%) of patients or mean } \pm \text { standard deviation values. Differences in parameters between groups were analyzed using one-way ANOVA for continuous variables, } \\
\text { or Kruskal-Wallis ANOVA test and a Dunn-Bonferroni test for post hoc comparisons for categorical variables. } \\
\text { BMI: Body mass index; M: Microfracture and saline; MS: Microfracture and sodium hyaluronic acid; MSR: Microfracture, sodium hyaluronic acid and Re-Join }{ }^{\circledR} ; \text { SF-36: Short-form } \\
36 \text { (quality of life); VAS: Visual analog scale; WOMAC: Western Ontario and McMaster University Osteoarthritis Index. }\end{array}$} \\
\hline
\end{tabular}

and pain relieved spontaneously; skin erythema around the treated knees was reported by two patients and was resolved without medication.

Complications not related to the procedure were also identified and recorded, including urinary tract, respiratory and dental infections, diarrhea, cardiac failure, urinary retention, confusional state and headache.

\section{Changes in WOMAC Scores}

Figure 2A, B and Table 4 show the WOMAC scores for patients with medial femoro-tibial condylar cartilage defects. Pretreatment WOMAC total scores were nearly identical, with a small nonsignificant difference observed among M, MS and MSR groups ( $40.2 \pm 12.9$ vs $40.9 \pm 6.26$ vs $45.8 \pm 10.2$, respectively; $\mathrm{p}=0.419)$ (Table 1 ). At 3 months after treatment, the WOMAC total scores in all groups improved, but the difference among three groups were nonsignificant $(\mathrm{p}=0.625)$. At 6 months post-treatment, the differences in improvement in WOMAC 

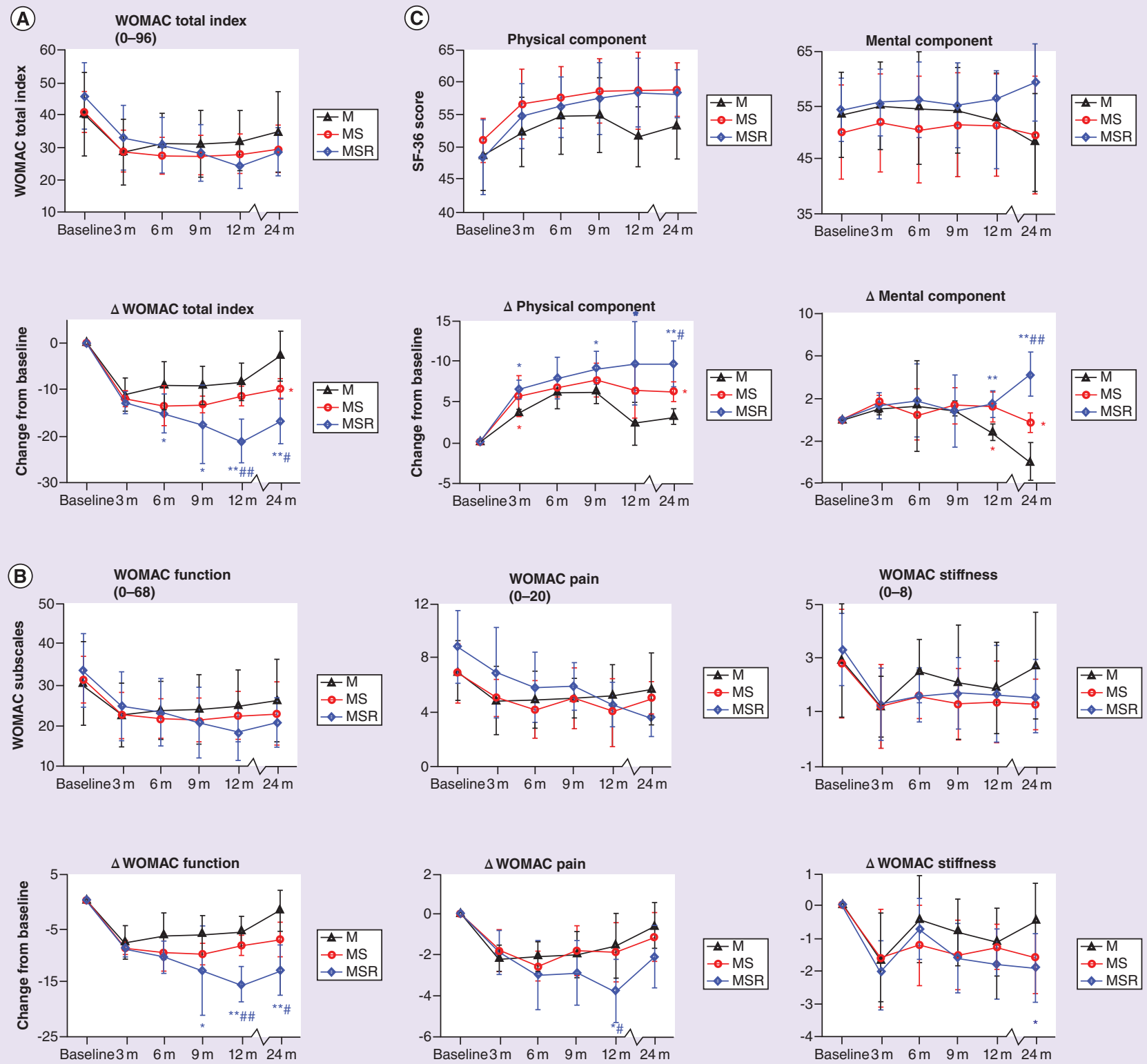

Figure 2. Changes in Western Ontario and McMaster University Osteoarthritis Index and Short-form 36 (quality of life) scores after treatment. Medial femoro-tibial condyle defect. (A \& B) The WOMAC total index and WOMAC subscales. A tendency of improvement in WOMAC total index and all the subscales was shown in the first year in all groups. However, after 1 year, the scores in M group declined, while in the other two groups, the WOMAC scores continued to improve. The changes in WOMAC score among the treatment groups were similar at month 3 and became significant later. (C) SF-36 score. Improvement of SF-36 score was seen in the MS and MSR groups. In the M group, a similar tendency was seen during the first year, while SF-36 score declined at month 24 . Differences were analyzed by the one-way analysis of variance (one-way ANOVA) test and a Dunn-Bonferroni test for post hoc comparisons. p-values were calculated only for comparisons between $\Delta$ values.

${ }^{*} \mathrm{p}<0.05$ and ${ }^{* *} \mathrm{p}<0.001$ vs $\mathrm{M}$ group, ${ }^{\#} \mathrm{p}<0.05$ and ${ }^{\# \#} \mathrm{p}<0.001$ vs MS group.

$\Delta$ : Change from baseline; M: Microfracture and saline; MS: Microfracture and sodium hyaluronic acid; MSR: Microfracture, sodium hyaluronic acid and Re-Join ${ }^{\circledR}$ injections; SF-36: Short-form 36 (quality of life); WOMAC: Western Ontario and McMaster University Osteoarthritis Index. 


\begin{tabular}{|c|c|c|c|c|c|c|c|c|c|}
\hline \multirow[t]{2}{*}{ Treatment Group } & \multicolumn{3}{|c|}{ 0-6 Months } & \multicolumn{3}{|c|}{ 6-12 Months } & \multicolumn{3}{|c|}{ After 12 Months } \\
\hline & $M(n=9)$ & MS $(n=9)$ & MSR $(n=10)$ & $M(n=9)$ & MS $(n=8)$ & $\operatorname{MSR}(n=9)$ & $M(n=7)$ & MS $(n=8)$ & MSR $(n=8)$ \\
\hline AEs & 10 & 11 & 9 & 6 & 5 & 3 & 4 & 4 & 1 \\
\hline Patients with AEs & 4 & 4 & 5 & 3 & 4 & 3 & 3 & 3 & 1 \\
\hline Patients with serious AEs & - & - & - & - & - & - & - & - & - \\
\hline \multicolumn{10}{|l|}{ Infections } \\
\hline - Urinary tract infection & 1 & - & - & 1 & - & - & - & 1 & - \\
\hline - Respiratory infection & 2 & - & 1 & 1 & - & 1 & 1 & - & 1 \\
\hline Dental infection & - & 1 & - & - & 1 & - & - & 1 & - \\
\hline \multicolumn{10}{|l|}{ Musculoskeletal disorders } \\
\hline - Joint effusion/swelling, treated knee & 2 & 1 & 2 & - & - & 1 & - & - & - \\
\hline - Sciatic pain & 1 & 2 & 2 & 1 & - & - & - & - & - \\
\hline - Low back pain & 2 & 1 & 1 & 2 & 1 & - & 1 & 1 & - \\
\hline - Skin erythema around the treated knee & - & 1 & 1 & 1 & - & 1 & - & - & - \\
\hline \multicolumn{10}{|l|}{ Gastrointestinal disorders } \\
\hline - Diarrhea & - & 1 & - & - & 1 & - & 1 & - & - \\
\hline \multicolumn{10}{|l|}{ Cardiovascular disorders } \\
\hline - Pulmonary embolism & - & - & - & - & - & - & - & - & - \\
\hline - Cardiac failure & - & 1 & - & - & - & - & - & - & - \\
\hline - Myocardial infarction & - & - & - & - & - & - & - & - & - \\
\hline - Acute cardiac arrhythmia & - & - & - & - & - & - & - & - & - \\
\hline \multicolumn{10}{|l|}{ Urinary disorders } \\
\hline - Urinary retention & 1 & 2 & 1 & - & - & - & - & - & - \\
\hline \multicolumn{10}{|l|}{ Neurological disorders } \\
\hline - Cerebrovascular accident & - & - & - & - & - & - & - & - & - \\
\hline - Confusional state & 1 & - & - & - & 1 & - & - & 1 & - \\
\hline - Headache & - & 1 & 1 & - & 1 & - & 1 & - & - \\
\hline
\end{tabular}

total scores among M, MS and MSR groups were significant ( $p=0.016$, M vs MS, $p=0.116$; MS vs MSR, $\mathrm{p}=1.000 ; \mathrm{M}$ vs MSR; $\mathrm{p}=0.017)$ and the differences became more pronounced after 12 months of monitoring ( $\mathrm{p}<0.001$; $\mathrm{M}$ vs MS, $\mathrm{p}=0.366$; MS vs MSR, $\mathrm{p}<0.001$; $\mathrm{M}$ vs MSR; $\mathrm{p}<0.001$ ). Significant differences in the WOMAC function subscales among the three groups were also observed at 9 months $(\mathrm{p}=0.034), 12$ months $(\mathrm{p}<0.001)$ and 24 months $(\mathrm{p}<0.001)$ after surgery. More importantly, the improvement in WOMAC scores in the MS group and MSR group maintained, while in the M group, the clinical effect nearly disappeared. The improvement in WOMAC stiffness was bigger in the MS and MSR group, but the difference among three groups were nonsignificant except at months $24(\mathrm{p}=0.044)$. Post hoc comparisons showed that the difference was between $\mathrm{M}$ and MSR group ( $\mathrm{M}$ vs MSR, $-0.429 \pm 1.13$ vs $-1.89 \pm 1.05 ; \mathrm{p}=0.048$ ).

\section{Changes in SF-36 Scores}

Figure 2C and Table 4 show the SF-36 scores for patients with medial femoro-tibial condylar cartilage defects. The differences in baseline physical scales $(p=0.500)$ and mental scales were nonsignificant $(p=0.466)$. After treatment, the physical scales in all groups increased compared with the pretreatment scores, and the improvement among the groups was significant $(\mathrm{p}<0.05)$ at all the follow-ups except at month $6(\mathrm{p}=0.165)$. Post hoc analysis showed that the improvement was significant better in MSR group, especially at month 12 and 24. Mental scales showed a similar tendency of improvement for all the treatment groups during the 2-year follow-up, but in the $\mathrm{M}$ group, mental scores were worse than baseline at month 12 and 24. The differences in SF-36 mental improvement were significantly at months 12 and $24(\mathrm{p}<0.001)$ and were nonsignificant at months 6,9 and $12(\mathrm{p}>0.05)$. Post hoc analysis showed that the improvement was significantly better in MSR group at month 12 (M vs MS, $-1.22 \pm 0.833$ vs $1.25 \pm 1.49, \mathrm{p}=0.001 ; \mathrm{M}$ vs MSR, $-1.22 \pm 0.833$ vs $1.44 \pm 1.33 ; \mathrm{p}<0.001)$, and at month 
Table 4. Clinical outcomes for patients with medial femoro-tibial condylar cartilage defect.

\begin{tabular}{|c|c|c|c|c|}
\hline & M group & MS group & MSR group & p-value \\
\hline \multicolumn{5}{|l|}{ WOMAC score } \\
\hline \multicolumn{5}{|l|}{ Total index } \\
\hline$\Delta, 3$ months & $-11.6 \pm 3.69(n=10)$ & $-12.1 \pm 1.79(n=10)$ & $-12.8 \pm 2.44(n=10)$ & 0.625 \\
\hline$\Delta, 6$ months & $-9.00 \pm 4.94(n=10)$ & $-13.4 \pm 4.43(n=10)$ & $-15.1 \pm 4.18^{\dagger}(n=10)$ & 0.016 \\
\hline$\Delta, 9$ months & $-9.10 \pm 4.20(n=10)$ & $-13.2 \pm 1.81(n=10)$ & $-17.4 \pm 9.03^{\dagger}(n=10)$ & 0.014 \\
\hline$\Delta, 12$ months & $-8.33 \pm 4.06(n=9)$ & $-11.4 \pm 2.20(n=8)$ & $-21.0 \pm 4.80 \pm(n=9)$ & $<0.001$ \\
\hline$\Delta, 24$ months & $-2.86 \pm 5.40(n=7)$ & $-9.86 \pm 2.34^{\dagger}(n=7)$ & $-16.8 \pm 4.97^{\ddagger \S}(n=9)$ & $<0.001$ \\
\hline \multicolumn{5}{|l|}{ Function subscale } \\
\hline$\Delta, 3$ months & $-7.70 \pm 3.16(n=10)$ & $-8.70 \pm 1.42(n=10)$ & $-8.90 \pm 1.59(n=10)$ & 0.438 \\
\hline$\Delta, 6$ months & $-6.50 \pm 4.33(n=10)$ & $-9.60 \pm 3.50(n=10)$ & $-10.4 \pm 2.99(n=10)$ & 0.057 \\
\hline$\Delta, 9$ months & $-6.30 \pm 3.59(n=10)$ & $-9.90 \pm 2.08(n=10)$ & $-12.9 \pm 8.25^{\dagger}(n=10)$ & 0.034 \\
\hline$\Delta, 12$ months & $-5.67 \pm 2.87(n=9)$ & $-8.25 \pm 1.91(n=8)$ & $-15.4 \pm 3.36^{\ddagger} \mathbb{I}(n=9)$ & $<0.001$ \\
\hline$\Delta, 24$ months & $-1.86 \pm 3.89(n=7)$ & $-7.14 \pm 3.18(n=7)$ & $-12.8 \pm 4.74 \mp \S(n=9)$ & $<0.001$ \\
\hline \multicolumn{5}{|l|}{ Pain subscale } \\
\hline$\Delta, 3$ months & $-2.20 \pm 0.632(n=10)$ & $-1.80 \pm 1.03(n=10)$ & $-1.90 \pm 1.10(n=10)$ & 0.621 \\
\hline$\Delta, 6$ months & $-2.10 \pm 0.738(n=10)$ & $-2.60 \pm 0.699(n=10)$ & $-3.00 \pm 1.70(n=10)$ & 0.229 \\
\hline$\Delta, 9$ months & $-2.00 \pm 1.15(n=10)$ & $-1.80 \pm 1.23(n=10)$ & $-2.90 \pm 1.59(n=10)$ & 0.167 \\
\hline$\Delta, 12$ months & $-1.56 \pm 1.51(n=9)$ & $-1.88 \pm 1.46(n=8)$ & $-3.78 \pm 1.56^{\dagger \S}(n=9)$ & 0.010 \\
\hline$\Delta, 24$ months & $-0.571 \pm 1.13(n=7)$ & $-1.14 \pm 1.21(n=7)$ & $-2.11 \pm 1.54(n=9)$ & 0.088 \\
\hline \multicolumn{5}{|l|}{ Stiffness subscale } \\
\hline$\Delta, 3$ months & $-1.70 \pm 1.49(n=10)$ & $-1.60 \pm 1.51(n=10)$ & $-2.00 \pm 0.943(n=10)$ & 0.787 \\
\hline$\Delta, 6$ months & $-0.400 \pm 1.35(n=10)$ & $-1.20 \pm 1.23(n=10)$ & $-0.700 \pm 0.949(n=10)$ & 0.064 \\
\hline$\Delta, 9$ months & $-0.800 \pm 1.03(n=10)$ & $-1.50 \pm 1.08(n=10)$ & $-1.60 \pm 1.07(n=10)$ & 0.205 \\
\hline$\Delta, 12$ months & $-1.11 \pm 1.05(n=9)$ & $-1.25 \pm 0.707(n=8)$ & $-1.78 \pm 1.09(n=9)$ & 0.332 \\
\hline$\Delta, 24$ months & $-0.429 \pm 1.13(n=7)$ & $-1.57 \pm 1.13(n=7)$ & $-1.89 \pm 1.05^{\dagger}(n=9)$ & 0.044 \\
\hline \multicolumn{5}{|l|}{ SF-36 score } \\
\hline \multicolumn{5}{|c|}{ Physical component } \\
\hline$\Delta, 3$ months & $3.61 \pm 0.381(n=10)$ & $5.65 \pm 2.50^{\dagger}(n=10)$ & $6.44 \pm 1.21^{\dagger}(n=10)$ & 0.002 \\
\hline$\Delta, 6$ months & $6.12 \pm 1.97(n=10)$ & $6.62 \pm 1.19(n=10)$ & $7.83 \pm 2.60(n=10)$ & 0.165 \\
\hline$\Delta, 9$ months & $6.20 \pm 1.59(n=10)$ & $7.59 \pm 2.10(n=10)$ & $9.10 \pm 2.09^{\dagger}(n=10)$ & 0.009 \\
\hline$\Delta, 12$ months & $2.23 \pm 2.66(n=9)$ & $6.30 \pm 3.42(n=8)$ & $9.62 \pm 5.15^{\dagger}(n=9)$ & 0.002 \\
\hline$\Delta, 24$ months & $2.94 \pm 0.824(n=7)$ & $6.19 \pm 1.26^{\dagger}(n=7)$ & $9.57 \pm 2.86^{\ddagger \S}(n=9)$ & $<0.001$ \\
\hline \multicolumn{5}{|c|}{ Mental component } \\
\hline$\Delta, 3$ months & $1.70 \pm 0.483(n=10)$ & $1.70 \pm 0.675(n=10)$ & $1.30 \pm 1.25(n=10)$ & 0.501 \\
\hline$\Delta, 6$ months & $1.30 \pm 4.32(n=10)$ & $0.500 \pm 2.46(n=10)$ & $1.80 \pm 3.49(n=10)$ & 0.708 \\
\hline$\Delta, 9$ months & $0.900 \pm 0.568(n=10)$ & $1.30 \pm 1.77(n=10)$ & $0.800 \pm 3.46(n=10)$ & 0.873 \\
\hline$\Delta, 12$ months & $-1.22 \pm 0.833(n=9)$ & $1.25 \pm 1.49^{\dagger}(n=8)$ & $1.44 \pm 1.33^{\ddagger}(n=9)$ & $<0.001$ \\
\hline$\Delta, 24$ months & $-4.00 \pm 1.83(n=7)$ & $-0.286 \pm 0.951^{\dagger}(n=7)$ & $4.33 \pm 2.12^{\ddagger}(n=9)$ & $<0.001$ \\
\hline \multicolumn{5}{|c|}{$\begin{array}{l}\text { Data are expressed as mean } \pm \text { standard deviation values. Differences in parameters between groups were analyzed by the one-way analysis of variance (one-way ANOVA) test } \\
\text { and a Dunn-Bonferroni test for post hoc comparisons. } \\
\dagger_{p}<0.05 \text {. } \\
\ddagger_{p}<0.001 \text { vs M group. } \\
\S_{p}<0.05 \text {. } \\
\mathbb{I}_{p}<0.001 \text { vs MS group. } \\
\Delta: \text { Change from baseline: M: Microfracture and saline; MS: Microfracture and sodium hyaluronic acid injections; MSR: Microfracture, sodium hyaluronic acid, and Re-Join }{ }^{\circledR} \\
\text { injections; WOMAC: Western Ontario and McMaster University Osteoarthritis Index. }\end{array}$} \\
\hline
\end{tabular}

24 (M vs MS, $-4.00 \pm 1.83$ vs $-0.286 \pm 0.951, \mathrm{p}<0.001 ; \mathrm{M}$ vs $\mathrm{MSR},-4.00 \pm 1.83$ vs $4.33 \pm 2.12, \mathrm{p}<0.001$; MS vs MSR, $-0.286 \pm 0.951$ vs $4.33 \pm 2.12 ; \mathrm{p}<0.001)$. 
Table 5. Arthroscopic and radiological outcomes for patients with femoro-tibial condylar cartilage defect.

\begin{tabular}{|c|c|c|c|c|}
\hline $\begin{array}{l}\text { Arthroscopic and radiological } \\
\text { parameters }\end{array}$ & M group & MS group & MSR group & p-value \\
\hline \multicolumn{5}{|l|}{ Arthroscopic defect size $\left(\mathrm{mm}^{2}\right)$} \\
\hline - Baseline & $\mathrm{n}=10$ & $\mathrm{n}=10$ & $\mathrm{n}=10$ & \\
\hline - Femoral condyle & $652.4 \pm 121.3$ & $585.3 \pm 102.1$ & $564.6 \pm 133.8$ & 0.325 \\
\hline - Tibial condyle & $372.3 \pm 124.3$ & $333.9 \pm 136.5$ & $333.7 \pm 139.0$ & 0.882 \\
\hline - Month 6 & $n=9$ & $n=9$ & $\mathrm{n}=10$ & \\
\hline - Femoral condyle & $671.7 \pm 92.7$ & $513.9 \pm 127.0$ & $406.8 \pm 134.8$ & \\
\hline - Tibial condyle & $362.4 \pm 123.1$ & $270.0 \pm 148.8$ & $239.8 \pm 136.9$ & \\
\hline \multicolumn{5}{|l|}{$\Delta$, Month 6} \\
\hline - Femoral condyle & $19.2 \pm 45.8$ & $-71.4 \pm 45.7^{\dagger}$ & $-157.8 \pm 43.5^{\ddagger \S}$ & $<0.001$ \\
\hline - Tibial condyle & $-9.9 \pm 42.4$ & $-63.9 \pm 44.6^{\dagger}$ & $-93.9 \pm 36.9^{\ddagger}$ & 0.001 \\
\hline \multicolumn{5}{|l|}{ MRI defect size $\left(\mathrm{mm}^{2}\right)$} \\
\hline - Baseline & $\mathrm{n}=10$ & $\mathrm{n}=10$ & $\mathrm{n}=10$ & \\
\hline - Femoral condyle & $424.6 \pm 71.3$ & $382.5 \pm 63.6$ & $391.6 \pm 63.5$ & 0.341 \\
\hline - Tibial condyle & $232.2 \pm 78.0$ & $210.2 \pm 85.4$ & $225.3 \pm 72.8$ & 0.817 \\
\hline - Month 6 & $\mathrm{n}=10$ & $\mathrm{n}=10$ & $\mathrm{n}=10$ & \\
\hline - Femoral condyle & $405.3 \pm 68.5$ & $337.5 \pm 67.3$ & $285.9 \pm 70.5$ & \\
\hline - Tibial condyle & $227.8 \pm 72.5$ & $180.7 \pm 82.1$ & $167.2 \pm 81.9$ & \\
\hline \multicolumn{5}{|l|}{$\Delta$, Month 6} \\
\hline - Femoral condyle & $-19.3 \pm 29.9$ & $-45.0 \pm 19.4$ & $-105.7 \pm 38.3^{\ddagger} \mathbb{I}$ & $<0.001$ \\
\hline - Tibial condyle & $-4.4 \pm 13.2$ & $-29.5 \pm 19.2^{\dagger}$ & $-58.1 \pm 20.7 \ddagger \S$ & $<0.001$ \\
\hline \multicolumn{5}{|l|}{ Cartilage volume $\left(\mathrm{mm}^{3}\right)$} \\
\hline - Baseline & $n=10$ & $n=10$ & $\mathrm{n}=10$ & \\
\hline - Femoral condyle & $3550.7 \pm 317.0$ & $3686.1 \pm 311.3$ & $3486.9 \pm 364.0$ & 0.403 \\
\hline - Tibial condyle & $1382.3 \pm 147.1$ & $1483.0 \pm 181.3$ & $1375.9 \pm 121.5$ & 0.228 \\
\hline - Month 6 & $\mathrm{n}=10$ & $\mathrm{n}=10$ & $\mathrm{n}=10$ & \\
\hline - Femoral condyle & $3593.9 \pm 393.2$ & $3856.4 \pm 304.0$ & $3841.7 \pm 424.6$ & \\
\hline - Tibial condyle & $1468.5 \pm 168.0$ & $1550.7 \pm 223.5$ & $1560.9 \pm 151.9$ & \\
\hline \multicolumn{5}{|l|}{$\Delta$, Month 6} \\
\hline - Femoral condyle & $48.2 \pm 141.6$ & $170.3 \pm 119.4$ & $354.8 \pm 110.3^{\ddagger \S}$ & $<0.001$ \\
\hline - Tibial condyle & $86.2 \pm 88.4$ & $67.7 \pm 71.2$ & $185.0 \pm 57.6^{\dagger \S}$ & 0.003 \\
\hline \multicolumn{5}{|c|}{$\begin{array}{l}\text { Data are expressed as mean } \pm \text { standard deviation values. Differences in parameters between groups were analyzed by the one-way analysis of variance (one-way ANOVA) test } \\
\text { and a Dunn-Bonferroni test for post hoc comparisons. Mann-Whitney U test for nonparametric variables within the group. } \\
\dagger_{p}<0.05 \text {. } \\
\ddagger p<0.001 \text { vs M group. } \\
s_{p}<0.05 \text {. } \\
\mathbb{I}_{p}<0.001 \text { vs MS group. } \\
\Delta: \text { Change from baseline; M: Microfracture and saline; MRI: Magnetic resonance imaging; MS: Microfracture and sodium hyaluronic acid; MSR: Microfracture, sodium hyaluronic } \\
\text { acid and Re-Join }{ }^{\circledR} \text { injections; WOMAC: Western Ontario and McMaster University Osteoarthritis Index. }\end{array}$} \\
\hline
\end{tabular}

\section{Arthroscopic evaluation of the cartilage defect}

All the patients who participated in the clinical trial agreed to undergo a second-look arthroscopy at enrollment. However, at month 6 only 28 out of 30 patients underwent second-look arthroscopy. Representative arthroscopic findings at baseline and 6 months after treatment are shown in Figure 3, and the detailed information is shown in Table 5 .

In medial femoral condyles, large areas of cartilage defects could be seen in all treatment groups at baseline. At second-look arthroscopy, only small cartilage islands formed in the M group. In the MS group, the defect area was covered with white cartilage, but the defect was not fully filled and the edge between normal cartilage and regenerated cartilage could be clearly seen. In the MSR group, the defect at baseline was fully filled with smooth, white hyaline-like cartilage, which integrated well with normal cartilage. The regenerated cartilage felt firm with a probe and no loose body, hypertrophy, or abnormal calcification was identified. The size of the cartilage defect is shown in the upper right graph in Figure 4B. The reduction in the cartilage defect in the M group was 
(A)

$\mathrm{M}$

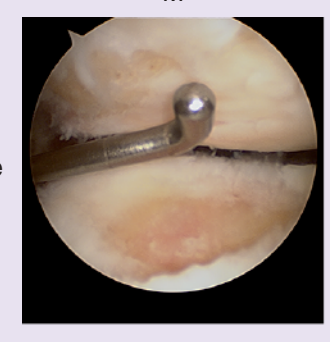

Baseline

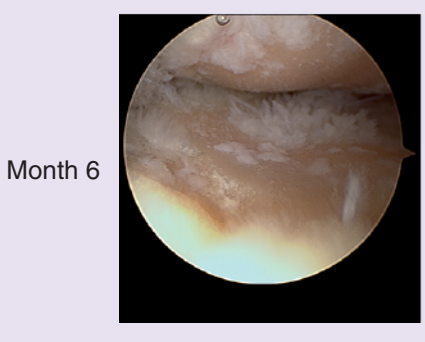

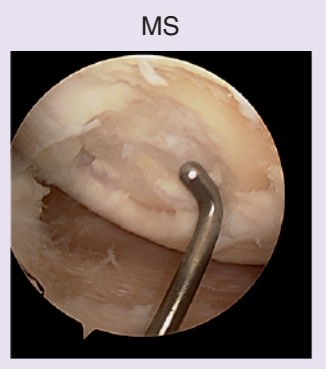
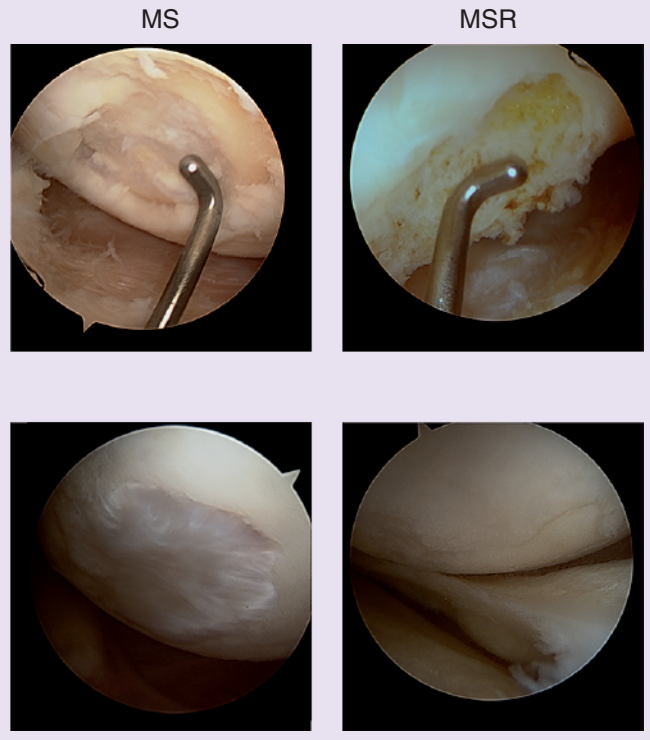

(B) Medial femoral condyle
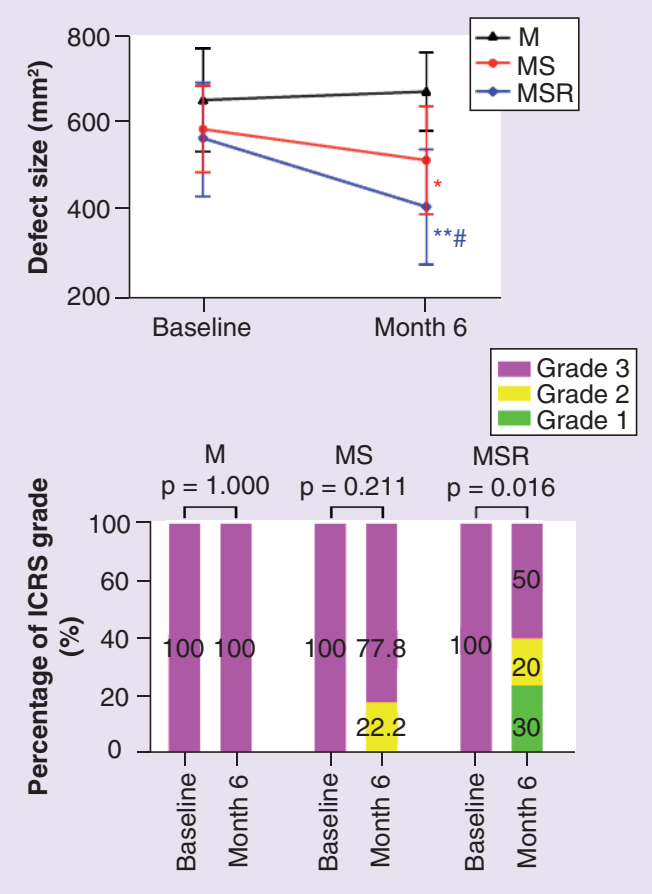
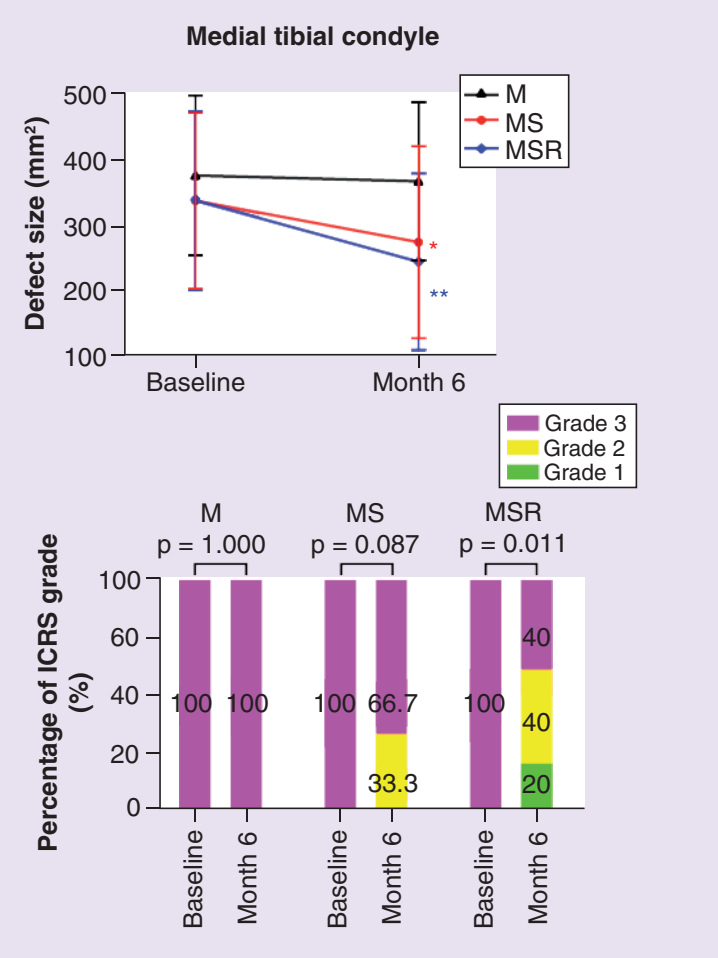

Figure 3. Arthroscopic evaluation of cartilage regeneration. Medial femoro-tibial condyle defect. (A) At baseline, large cartilage defects in medial femoral condyles can be seen in all treatment groups. In the M group, while small cartilage islands formed, most cartilage defects are not covered 6 months after treatment. In the MS group, at 6 months, newly formed white cartilage is visible, while the defect is not fully filled and the edge can be seen clearly. In the MSR group, most of the defect area is covered with thick, smooth, hyaline-like cartilage 6 months after treatment. (B) In the MSR group, the ICRS grade of the articular cartilage significantly improved (femoral, $p=0.016$; Tibial, $p=0.011$ ), while no significant change was found in the $M$ (femoral, $p=1.000 ;$ tibial, $p=1.000$ ) and MS (femoral, $\mathrm{p}=0.211$; tibial, $\mathrm{p}=0.087$ ) groups.

${ }^{*} \mathrm{p}<0.05$ and ${ }^{* *} \mathrm{p}<0.001$ vs $\mathrm{M}$ group, ${ }^{\#} \mathrm{p}<0.05$ and ${ }^{\# \#} \mathrm{p}<0.001$ vs MS group. Differences in parameters between groups were analyzed by the one-way analysis of variance (one-way ANOVA) test and a Dunn-Bonferroni test for post hoc comparisons. The change in ICRS grade in each group were analyzed by the Mann-Whitney $U$ test.

$\Delta$ : Change from baseline; ICRS: International Cartilage Repair Society; M: Microfracture and saline; MS: Microfracture and sodium hyaluronic acid; MSR: Microfracture, sodium hyaluronic acid and Re-Join $\AA$ injections. 


\begin{tabular}{|c|c|c|c|c|c|c|c|c|c|}
\hline \multirow[t]{2}{*}{ Histological parameter } & \multicolumn{3}{|c|}{$M(n=9)$} & \multicolumn{3}{|c|}{ MS $(n=9)$} & \multicolumn{3}{|c|}{$\operatorname{MSR}(n=10)$} \\
\hline & Baseline & Month 6 & p-value & Baseline & Month 6 & p-value & Baseline & Month 6 & p-value \\
\hline Tissue morphology & $33.2 \pm 19.2$ & $37.6 \pm 16.9$ & 0.618 & $33.9 \pm 17.6$ & $43.3 \pm 20.8$ & 0.313 & $35.9 \pm 18.3$ & $48.7 \pm 23.2$ & 0.129 \\
\hline Matrix staining & $27.0 \pm 21.2$ & $31.1 \pm 23.2$ & 0.700 & $32.6 \pm 18.5$ & $40.8 \pm 18.1$ & 0.355 & $28.3 \pm 14.0$ & $38.7 \pm 14.5$ & 0.083 \\
\hline Cell morphology & $50.0 \pm 23.5$ & $50.3 \pm 22.0$ & 0.976 & $47.0 \pm 27.1$ & $46.2 \pm 26.5$ & 0.952 & $55.8 \pm 21.7$ & $57.6 \pm 21.4$ & 0.861 \\
\hline Chondrocyte clustering & $46.3 \pm 22.9$ & $48.2 \pm 25.4$ & 0.870 & $49.4 \pm 21.2$ & $57.7 \pm 20.8$ & 0.419 & $50.7 \pm 22.1$ & $66.9 \pm 16.2$ & 0.070 \\
\hline Surface architecture & $28.2 \pm 17.1$ & $28.2 \pm 16.0$ & 1.000 & $30.3 \pm 11.0$ & $51.9 \pm 12.8$ & 0.001 & $25.3 \pm 12.0$ & $68.4 \pm 16.4$ & $<0.001$ \\
\hline Basal integration & $55.8 \pm 20.6$ & $54.0 \pm 23.4$ & 0.867 & $52.8 \pm 30.8$ & $63.2 \pm 26.3$ & 0.450 & $56.4 \pm 14.0$ & $60.4 \pm 13.9$ & 0.563 \\
\hline Formation of the tidemark & $30.6 \pm 21.6$ & $33.1 \pm 24.9$ & 0.819 & $33.7 \pm 18.8$ & $31.8 \pm 17.7$ & 0.829 & $37.8 \pm 10.9$ & $41.8 \pm 15.0$ & 0.919 \\
\hline Subchondral bone/marrow fibrosis & $40.6 \pm 21.2$ & $40.7 \pm 22.3$ & 0.991 & $41.1 \pm 24.7$ & $43.9 \pm 25.6$ & 0.818 & $42.3 \pm 15.3$ & $35.3 \pm 18.7$ & 0.393 \\
\hline Inflammation & - & - & - & - & - & - & - & - & - \\
\hline Abnormal calcification/ossification & $56.2 \pm 25.8$ & $55.1 \pm 27.7$ & 0.931 & $58.1 \pm 14.7$ & $70.8 \pm 14.1$ & 0.081 & $60.1 \pm 24.2$ & $68.2 \pm 23.7$ & 0.392 \\
\hline Vascularization & $80.8 \pm 9.5$ & $78.1 \pm 11.1$ & 0.593 & $75.2 \pm 17.1$ & $72.9 \pm 15.6$ & 0.767 & $82.3 \pm 11.9$ & $89.8 \pm 6.0$ & 0.108 \\
\hline Surface/superficial assessment & $24.3 \pm 14.7$ & $26.3 \pm 17.7$ & 0.797 & $29.9 \pm 18.6$ & $45.3 \pm 20.4$ & 0.113 & $35.8 \pm 23.9$ & $68.8 \pm 20.7$ & 0.007 \\
\hline Mid/deep zone assessment & $29.1 \pm 16.2$ & $31.2 \pm 17.4$ & 0.794 & $25.0 \pm 13.5$ & $38.0 \pm 13.5$ & 0.058 & $21.7 \pm 8.0$ & $32.4 \pm 11.5$ & 0.054 \\
\hline Overall assessment & $28.1 \pm 19.1$ & $27.4 \pm 19.7$ & 0.943 & $27.7 \pm 16.4$ & $43.2 \pm 14.5$ & 0.049 & $32.0 \pm 21.6$ & $55.9 \pm 23.2$ & 0.030 \\
\hline
\end{tabular}

Data are presented as mean \pm standard deviation values. ICRS II is a histological scoring scheme designed to evaluate the quality of cartilage repair tissue in patients. Differences in parameters between groups were analyzed by the paired-samples t-test.

M: Microfracture and saline; MS: Microfracture and sodium hyaluronic acid; MSR: Microfracture, sodium hyaluronic acid and Re-Join ${ }^{\circledR}$ injections.

(A)
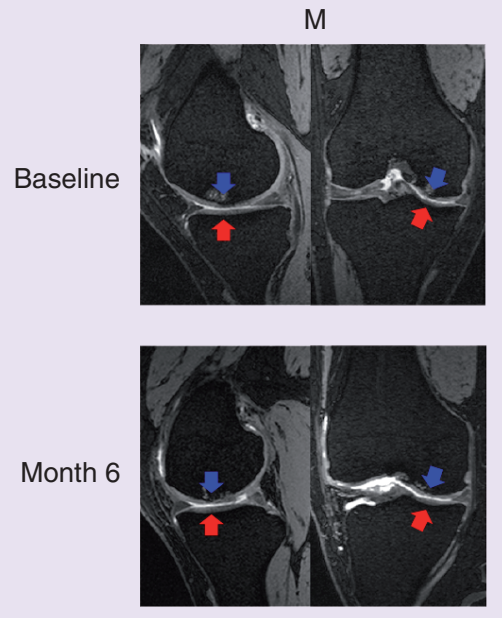

Femoral defect

Tibial defect
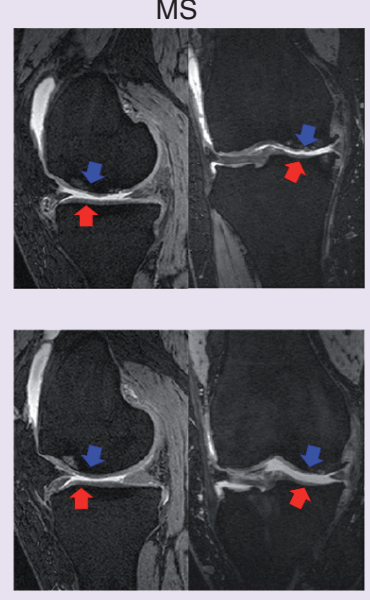
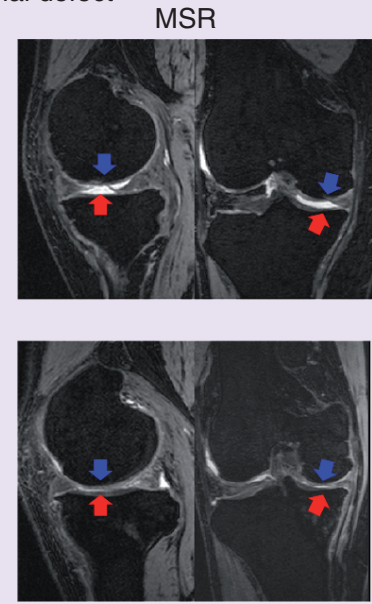

(B) Medial femoral condyle
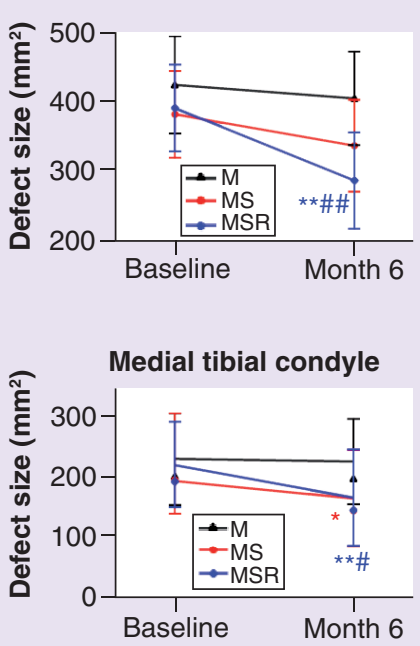

Figure 4. Magnetic resonance imaging evaluation of cartilage defect after treatment. Medial femoro-tibial condyle defect. (A) Sagittal and coronal MRIs of the medial femoral and tibial condyles before and 6 months after treatment. Cartilage defects in the medial femoral condyle and in the medial tibial condyle were identified as signal voids between the two condyles. In the M group, no substantial change was identified after treatment at months 6 . In the MS group, thin regenerated cartilage with small signal voids can be observed. In the MSR group, smoother and thicker regenerated cartilage was observed in the former defect areas. (B) Line chart showing cartilage defect size at baseline and month 6 .

${ }^{*} p<0.05$ and ${ }^{*} \mathrm{p}<0.001$ vs M group, $\# p<0.05$ and ${ }^{\# \#} \mathrm{p}<0.001$ vs MS group. Differences in parameters between groups were analyzed by the one-way analysis of variance (oneway ANOVA) test and a Dunn-Bonferroni test for post hoc comparisons.

M: Microfracture and saline; MRI: Magnetic resonance imaging; MS: Microfracture and sodium hyaluronic acid; MSR: Microfracture, sodium hyaluronic acid and Re-Join ${ }^{\circledR}$ injections. 
Table 7. Cartilage thickness before and after treatmentfor patients with femoro-tibial condylar cartilage defect.

\begin{tabular}{|c|c|c|c|c|}
\hline & M group & MS group & MSR group & $\mathrm{p}$-value \\
\hline \multicolumn{5}{|c|}{ Cartilage thickness (mm) } \\
\hline - Baseline & $0.52 \pm 0.28$ & $0.45 \pm 0.22$ & $0.57 \pm 0.30$ & 0.645 \\
\hline - Month 6 & $0.59 \pm 0.34$ & $0.85 \pm 0.37$ & $1.50 \pm 0.47$ & \\
\hline$-\Delta$, Month 6 & $0.07 \pm 0.37$ & $0.39 \pm 0.28$ & $0.93 \pm 0.55$ & 0.001 \\
\hline
\end{tabular}

$-19.9 \pm 45.8 \mathrm{~mm}^{2}$ in the medial femoral condyle and $9.9 \pm 42.4 \mathrm{~mm}^{2}$ in the medial tibial condyle; in the MS group, $71.4 \pm 45.7 \mathrm{~mm}^{2}$ in the medial femoral condyle and $63.9 \pm 44.6 \mathrm{~mm}^{2}$ in the medial tibial condyle; and in the MSR group, $157.8 \pm 43.5 \mathrm{~mm}^{2}$ in the medial femoral condyle and $93.9 \pm 36.9 \mathrm{~mm}^{2}$ in the medial tibial condyle. The difference between groups was significant $(\mathrm{p}<0.05)$. Post hoc analysis showed that the decrease in cartilage defect in MS and MSR groups was significantly larger than M group (femoral, M vs MSR, $19.2 \pm 45.8$ vs $-157.8 \pm 43.5, \mathrm{p}<0.001 ; \mathrm{M}$ vs MS, $19.2 \pm 45.8$ vs $-71.4 \pm 45.7, \mathrm{p}=0.001 ; \mathrm{MS}$ vs MSR, $-71.4 \pm 45.7$ vs $-157.8 \pm 43.5, \mathrm{p}=0.001$; tibial, $\mathrm{M}$ vs MSR, $-9.9 \pm 42.4$ vs $-93.9 \pm 36.9, \mathrm{p}<0.001 ; \mathrm{M}$ vs MS, $-9.9 \pm 42.4$ vs $-63.9 \pm 44.6, \mathrm{p}=0.031$; MS vs MSR, $-63.9 \pm 44.6$ vs $-93.9 \pm 36.9, \mathrm{p}=0.378)$. The detailed information is shown in Table 7. The ICRS grade of the cartilage defect significantly improved in the MSR group (femoral, $\mathrm{p}=0.016$; tibial, $\mathrm{p}=0.011$ ), while no significant change was observed in the M and MS ( $>>0.05$ ) groups (Figure 3B).

\section{MRI evaluation of the cartilage defect}

Representative MRI images of the cartilage defect before and 6 months after treatment are shown in Figure 4, and the change of the cartilage defect measured with MRI was shown in Table 5.

The reduction in cartilage defect in the $M$ group was $19.3 \pm 29.9 \mathrm{~mm}^{2}$ in the medial femoral condyle and $4.4 \pm 13.2 \mathrm{~mm}^{2}$ in the medial tibial condyle; in the MS group, $45.0 \pm 19.4 \mathrm{~mm}^{2}$ in the medial femoral condyle and $29.5 \pm 19.2 \mathrm{~mm}^{2}$ in the medial tibial condyle; and in the MSR group, $105.7 \pm 38.3 \mathrm{~mm}^{2}$ in the medial femoral condyle and $58.1 \pm 20.7 \mathrm{~mm}^{2}$ in the medial tibial condyle. The difference between groups was significant $(\mathrm{p}<0.001)$ and post hoc analysis show that compared with M and MS group, MSR group had a significantly larger reduction of cartilage defect ( $\mathrm{p}<0.05$ for all analysis). For patients with trochlear-patellar cartilage defects, the reduction of the cartilage defect in the $M$ group was $10.9 \pm 26.0 \mathrm{~mm}^{2}$ in the trochlea and $-4.5 \pm 19.8 \mathrm{~mm}^{2}$ in the patella; in the MS group, $46.3 \pm 21.9 \mathrm{~mm}^{2}$ in the trochlea and $55.0 \pm 20.7 \mathrm{~mm}^{2}$ in the patella; and in the MSR group, $85.8 \pm 23.4 \mathrm{~mm}^{2}$ in the trochlea and $117.9 \pm 24.8 \mathrm{~mm}^{2}$ in the patella. The difference between groups was significant $(\mathrm{p}<0.001)$. Post hoc analysis show that the reduction in cartilage defect of MSR group was significantly larger than the other two groups ( $\mathrm{p}<0.05$ for all analysis).

\section{Changes in the cartilage volume}

Representative 3D images of the cartilage at baseline and month 6 are shown in Figure 5. Detailed information is shown in Table 5.

For patients with cartilage defects in medial femoral and tibial condyles, the increase in the cartilage volume in the $M$ group was $48.2 \pm 141.6 \mathrm{~mm}^{3}$ in the medial femoral condyle and $86.2 \pm 88.4 \mathrm{~mm}^{3}$ in the medial tibial condyle; in the MS group, $170.3 \pm 119.4 \mathrm{~mm}^{3}$ in the medial femoral condyle and $67.7 \pm 71.2 \mathrm{~mm}^{3}$ in the medial tibial condyle; and in the MSR group, $304.8 \pm 64.0 \mathrm{~mm}^{3}$ in the medial femoral condyle and $185.0 \pm 57.6 \mathrm{~mm}^{3}$ in the medial tibial condyle. The difference between groups was significant $(\mathrm{p}<0.001$ for the femoral condyle and $\mathrm{p}=0.003$ for the tibial condyle). Post hoc analysis showed that the increase in cartilage volume of MSR group was significantly larger than the other two groups (femoral, M vs MS, $\mathrm{p}=0.069 ; \mathrm{M}$ vs MSR, $\mathrm{p}<0.001$; MS vs MSR, $\mathrm{p}=0.039$; tibial, $\mathrm{M}$ vs MS, $\mathrm{p}=1.000 ; \mathrm{M}$ vs MSR, $\mathrm{p}=0.017$; MS vs MSR, $\mathrm{p}=0.004)$.

\section{Histological outcomes}

Overall, the cartilage repair effect of the MSR group outperformed the other two groups. Representative histological images of the cartilage at baseline and month 6 are shown in Figure 6. Generally, biopsy specimens from the medial femoral condyles had only thin and rough articular cartilage before treatment (Figure 6). In the $M$ group, a combination of thin fibrocartilage and fibrotic tissue with a severely degraded surface is formed and integrated with the subchondral bone. Safranin O is stained mostly in the middle zones. Masson's trichrome and 
(A)
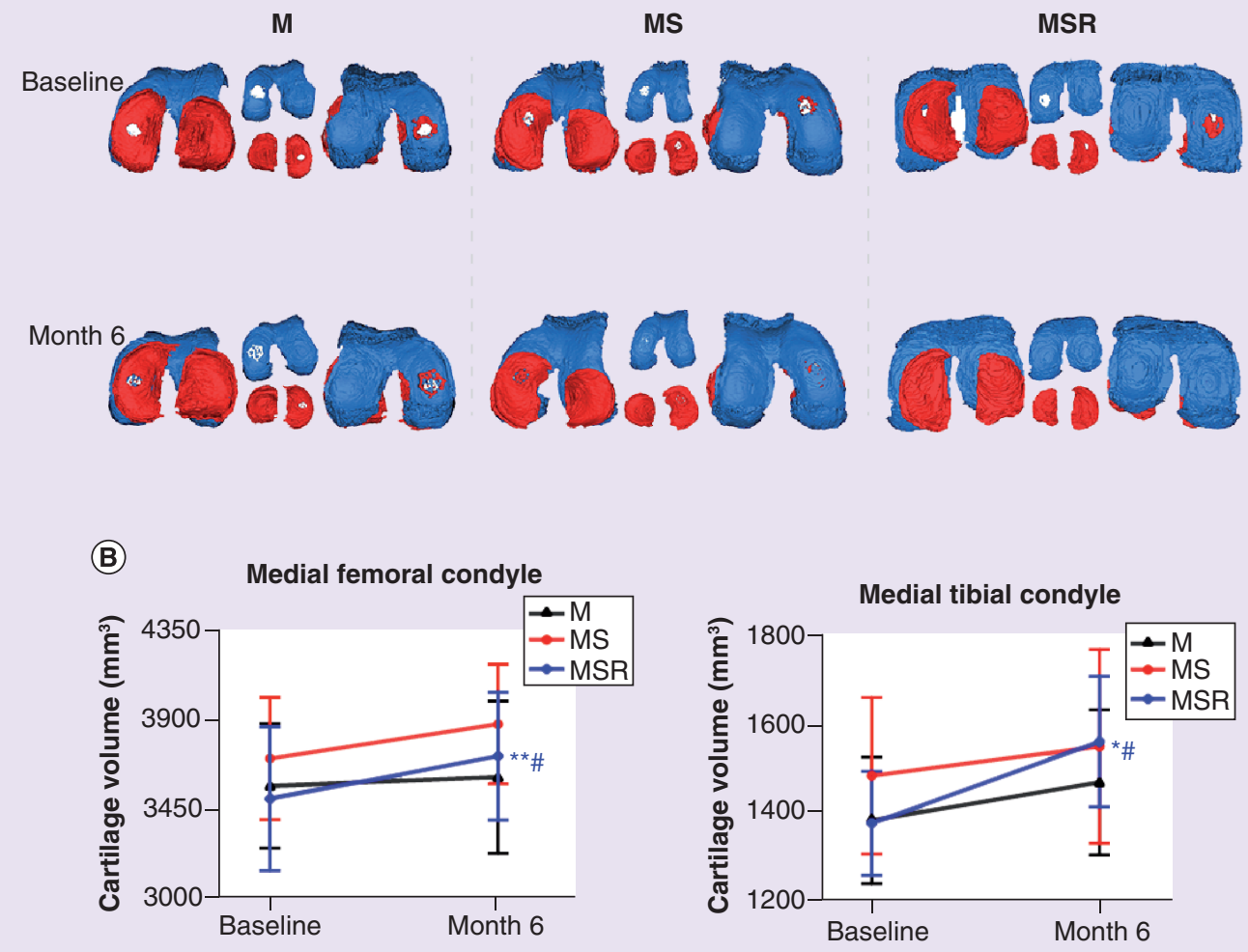

Figure 5. Changes in articular cartilage volume after treatment. Changes of the cartilage volume after treatment. (A) Changes in articular cartilage volume 6 months after treatment in the medial femoral condyle (blue) and in the medial tibial condyle (red) in the treatment groups. For each column, left is viewed from below, right is viewed from above, and the covered areas are shown in the middle. In the M group, the void seen at baseline exhibited little change at 6 months in the medial femoral and tibial condyles; while in the MS group, the void became smaller; and in the MSR group, the void was filled. (B) Line chart showing cartilage volume at baseline and month 6 .

${ }^{*} p<0.05$ and ${ }^{* *} p<0.001$ vs M group, ${ }^{\#} p<0.05$ and ${ }^{\# \#} p<0.001$ vs MS group. Differences in parameters between groups were analyzed by the one-way analysis of variance (one-way ANOVA) test and a Dunn-Bonferroni test for post hoc comparisons.

M: Microfracture and saline; MS: Microfracture and sodium hyaluronic acid; MSR: Microfracture, sodium hyaluronic acid and Re-Join ${ }^{\circledR}$ injections.

immunohistochemistry for type I and II collagen stain show that the regenerated tissue is fibrocartilage, composed of type II collagen and minimally type I collagen. Zonal structure or tide mark is not definite. In the MS group, 6 months after treatment, the cartilage defects were mostly completely filled with a fibrocartilaginous tissue. The surface is relatively smooth with little signs of degradation. Safranin $O$ is stained mostly in the lower half of the middle and the deep zones. Regenerated cartilage shows more positive Safranin $\mathrm{O}$ and collagen II staining. In the MSR group, a thick, hyaline-like cartilage with a smooth surface is regenerated and integrated with the subchondral bone 6 months after treatment. Safranin O and type II collagen is stained mostly in the lower half of the middle and the deep zones. Masson's trichrome staining and immunohistochemistry staining for type I collagen are less positive. Type II collagen staining is more positive. Zonal structure and tide mark are formed. Chondrocytes are flattened in the superficial zone and round in the middle and deep zones.

In the M group, the ICRS II scores did not change significantly after treatment. In the MS group, surface architecture and overall assessments improved significantly. While in the MSR group, after treatment the ICRS II scores improved significantly in four parameters: surface architecture, and surface, mid, and overall assessments (Table 6). 
(A)

M

(B)

Month 6
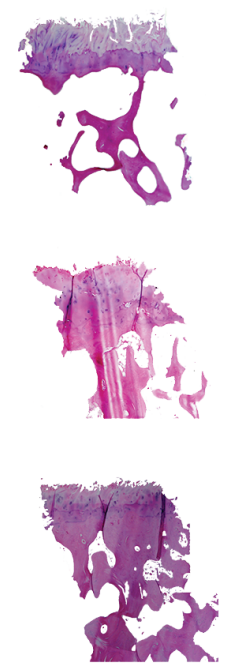

MS

Month 6

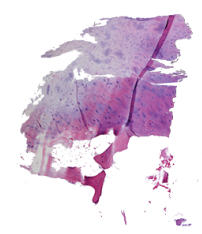

(C)

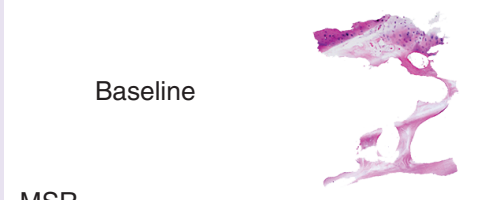

Month 6

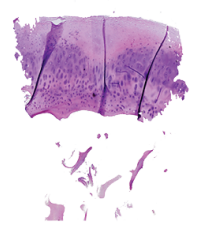

SOFG
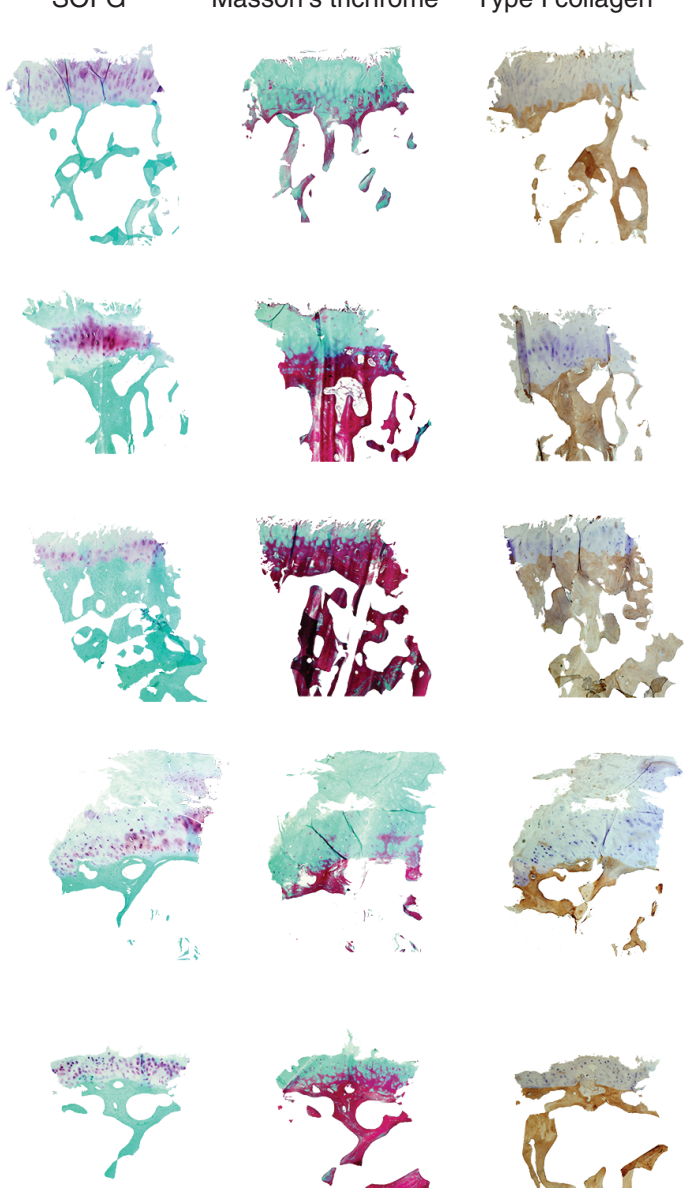

Type II collagen
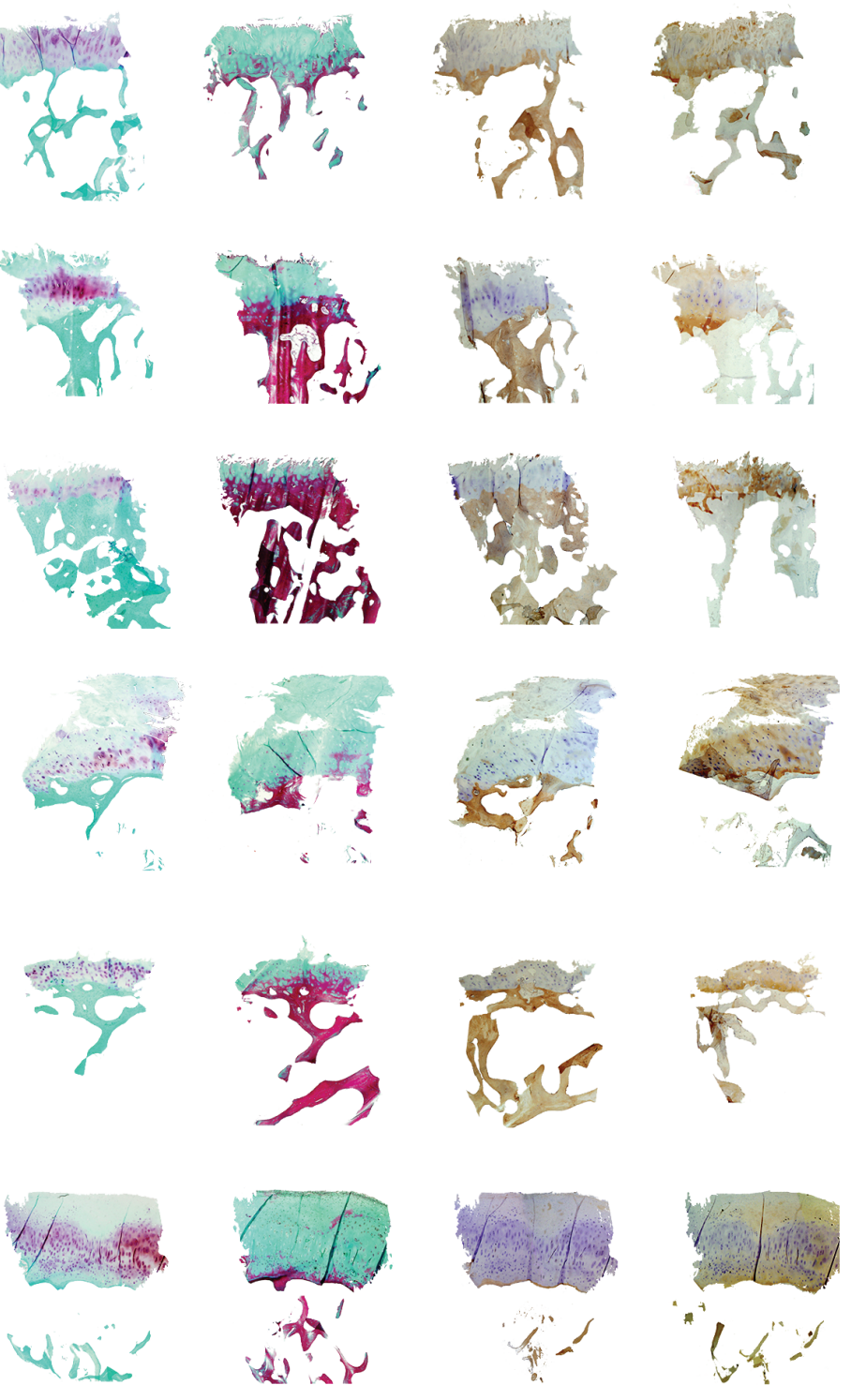

$\overline{200 \mu \mathrm{m}}$

Figure 6. Histological evaluation of articular cartilage of biopsy from the medial femoral condyle after treatment. Medial femoro-tibial condyle defect. (A) A typical biopsy sample from the medial femoral condyle of a patient in the M group at the baseline and 6 months after microfracture stained with H\&E, safranin O, Masson's trichrome and anti-type I and II collagen antibodies. Thin and rough articular cartilage is seen at the baseline. At month 6, a combination of thin fibrocartilage and fibrotic tissue is formed and integrated with the subchondral bone. The surface of the repair tissue is severely degraded. Safranin $\mathrm{O}$ is stained mostly in the middle zones. Masson's trichrome and immunohistochemistry for type I and II collagen stain show that the regenerated cartilage is fibrocartilaginous tissue, composed of type II collagen and minimally type I collagen. Zonal structure or tide mark is not definite. (B) A typical biopsy sample of a patient in the MS group. At baseline the articular cartilage is similar to (A). 6 months after treatment, the cartilage defects were mostly completely filled with a fibrocartilaginous tissue. The surface is relatively smooth with little signs of degradation. Safranin $\mathrm{O}$ is stained mostly in the lower half of the middle and the deep zones. Regenerated cartilage shows more positive Safranin O and collagen II staining. (C) A typical biopsy sample of a patient in the MSR group. At month 6, a thick, hyaline-like cartilage with a smooth surface is regenerated and integrated with the subchondral bone. Safranin $\mathrm{O}$ and type II collagen is stained mostly in the lower half of the middle and the deep zones. Masson's trichrome staining and immunohistochemistry staining for type I collagen are less positive. Type II collagen staining is more positive. Zonal structure and tide mark are formed. Chondrocytes are flattened in the superficial zone and round in the middle and deep zones.

H\&E: Hematoxylin-eosin; M: Microfracture and saline; MS: Microfracture and sodium hyaluronic acid; MSR: Microfracture, sodium hyaluronic acid and Re-Join ${ }^{\circledR}$ injections; SOFG: Safranin O/fast green. 
In the $\mathrm{M}$ group, the mean thickness of articular cartilage increased from $0.52 \pm 0.28 \mathrm{~mm}$ to $0.59 \pm 0.34$ after treatment; in the MS group, the mean thickness of cartilage was $0.45 \pm 0.22$ at baseline and $0.85 \pm 0.37$ after treatment; and in the MSR group, $0.57 \pm 0.30 \mathrm{~mm}^{3}$ before treatment and $1.50 \pm 0.47 \mathrm{~mm}^{3}$ at month 6 . The increase in cartilage thickness was statistically different between groups $(\mathrm{p}<0.001)$. Post hoc analysis showed that the increase in cartilage thickness of MSR group was significantly larger than the other two groups (M vs MS, $\mathrm{p}=0.362 ; \mathrm{M}$ vs MSR, $\mathrm{p}<0.001 ; \mathrm{MS}$ vs MSR, $\mathrm{p}=0.030$; Table 7).

\section{Discussion}

We conducted a Phase IIa proof-of-concept randomized, single-blind clinical trial to evaluate the safety and efficacy of Re-Join in combination with microfracture and HA in patients with knee cartilage defects. The findings support the hypothesis that microfracture in combination with HA and haMPCs will provide clinical improvement for patients with knee cartilage defects.

Baseline characteristics were well-matched, and study retention was adequate. Patients in MS and MSR group showed a significant response to treatment, for clinically evaluation with WOMAC scores and SF-36 scores, and for quantitative assessment of defect size and cartilage volume with MRI and arthroscopy. During the first 9 months post-treatment, the differences between groups were mostly nonsignificant. After 12 months, clinical improvement in the MSR group was significantly better than M and MS group in most scales. In the M group, clinical improvement was also observed, but only within 9 months follow-up, after which the benefits of microfracture were gradually lost, supporting the findings in published studies $[49,50]$ that arthroscopic microfracture reduce pain and improve knee function after the procedure, but these benefits only last for about 1 year. In the MS and MSR groups, clinical improvement also seemed to diminish after 1 year. But these outcome measures were still slightly improved at 24 months follow-up compared with baseline.

Published studies have reported significant relief of symptoms and improved joint function for knee OA after microfracture [6-10]. The mechanism is that penetration of the subchondral bone plate in defect areas lead to clot formation in the defect. This clot contains pluripotent, marrow-derived mesenchymal stem cells, which produce a fibrocartilage repair with varying amounts of type-II collagen content [51-58]. However, study groups in these studies were younger and with lower grade OA. In the present study, the patient population were 50-75 with moderate to severe (KL Grade 3) OA. The Ethics Committee required that patients in all treatment groups microfractured, therefore there were no cell-only group.

The WOMAC score decreased in all treatment groups shortly after treatment. We believe that in our scenario, older patients with cartilage defects in KL grade 3 knees can also benefit from microfracture, though the efficacy was not as much as in younger patients with lower grade OA. The differences in the improvement in WOMAC scores between groups were mostly nonsignificant during the first 3 months post-treatment. After 6 months, the difference in clinical improvement between treatment groups was significantly. Presumably HA and Re-Join did not add significantly to pain relief shortly following the procedure, and the difference in later follow-ups results in different durability of regenerative tissues of these treatments. These findings are consistent with a systematic review and meta-analysis which indicated that the efficacy of microfracture is only short term [50].

Compared with historical studies, the efficacy of Re-Join combined with HA and microfracture surgery in knee cartilage defects seems encouraging. For example, in a prospective open-label dose-escalating trial on injection of autologous ADSCs in a limited number of patients with knee OA, the WOMAC score in the high-dose group decreased by $21.7 \pm 15.4$ (0-96 scale) at month 6 [59]. In the present study, between baseline and 6 months, the WOMAC score decreased by $15.1 \pm 4.18$ for patients with medial femoro-tibial condyle cartilage defects, and $16.2 \pm 4.29$ for patients with trochlear-patellar defects. In a similar dose-escalating trial using ADSCs in patients with knee OA conducted by Jo et al., in the high-dose group in which the highest efficacy was found, the mean reduction in WOMAC score from baseline to 6 months was $54.2 \pm 5.2$ to $32.8 \pm 6.3$ [45]. Clinical results in the MSR group in our study are comparable to their results, and improvements were observed at later follow-ups.

The efficacy of microfracture and HA injection in knee OA in our study were also comparable with previous studies. In a randomized, double-blind, controlled trial using intra-articular HA for treating symptomatic knee OA, at 6 months, the WOMAC score decreased by $15.5 \pm 2.6$ [60]. In another randomized, double-blind, multicenter study comparing Artz to Durolane (nonanimal source HA) in treating knee OA, the WOMAC score decreased by 19.7 in the Artz group and 20.59 in the Durolane group at 18 weeks. At 26 weeks, the WOMAC score decreased by 20.43 in the Artz group and 21.26 in the Durolane group [61]. In our study, the WOMAC score in the MSR group decreased by $15.1 \pm 4.18$ at 24 weeks and further decreased by $17.4 \pm 9.08$ at 36 weeks after treatment. 
Treatments in this trial were only associated with several minor AEs, which were resolved spontaneously or with nonsteroidal anti-inflammatory drugs, and several AEs were not related to the procedures. These findings demonstrated that microfracture in combination with intra-articular injections of haMPCs and HA is a safe and effective procedure for improving clinical function in patients with knee cartilage defects.

The histological outcomes of the present study show that the treatment in patients with knee cartilage defects results in a fibrocartilage (M and MS groups) or a combination of fibrocartilage and hyaline-like cartilage (MSR group). The thickness of cartilage increased after treatment and cartilage-specific stains of the repair tissue were more intense than baseline, especially in MS and MSR groups. However, the regenerated tissues in the trial were not hyaline cartilage, but fibrocartilage or a combination of fibrocartilage and hyaline-like cartilage. The repair tissue had a better appearance than the cartilage at baseline, but did not reach the quality of normal hyaline. Also, for ethical reasons, we could only take biopsies with diameters of about $2 \mathrm{~mm}$, which do not allow judging the integration of the repair tissue with the adjacent articular cartilage, an important parameter for the long-term performance of the repair tissue. Given the above, the efficacy of MSR in cartilage regeneration cannot be proved, especially when we had histology data from a limited number of patients.

We recognize that there are limitations of the study. First, there was relatively small number of cases in this study. The limited number of patients included may explain why statistical significance was not reached for several scales. However, this study is designed to provide an impetus for further evaluation, it serves as a preliminary research to evaluate the potential role of intra-articular injection of haMPCs in knee cartilage defect, rather than provide definitive conclusions. Second, haMPCs were administered in combination with HA and microfracture. Due to ethical reasons, there were no cell-only group, therefore it was not able to fully evaluated the efficacy of haMPCs, and no specific conclusions can be drawn with regard to the effects of haMPCs. Third, although the WOMAC questionnaire is a validated instrument specifically designed to evaluate the condition of patients with $\mathrm{OA}$ of the knee and hip, it might not be specific enough for patients after arthroscopic microfracture and intra-articular injection of sodium HA and haMPCs. Researchers may need a specifically designed questionnaire for these patients.

\section{Conclusion}

In conclusion, this study confirms that intra-articular injections of haMPCs in conjunction with HA and microfracture improved clinical function in knee cartilage defect patients without inducing adverse events. Evaluation with WOMAC and SF-36 measures demonstrated the efficacy of the treatment. Radiological, arthroscopic and histological evaluation consistently confirmed that the treatment promoted cartilage defect decrease and cartilage regeneration, though cartilage regeneration efficacy of the treatment cannot be proved so far due to lack of evidence. These data demonstrated that intra-articular injections of haMPCs in conjunction with HA and microfracture are safe and effective for improving clinical functions for KL grade 3 patients with knee cartilage defects. The results are promising to encourage larger scales of randomized clinical trials to further verify the efficacy of haMPCs in conjunction with HA and microfracture in human knee cartilage defects.

\section{Translational perspective}

This study confirms the safety and clinical functional efficacy of haMPCs. HaMPCs are easy to collect for clinical application with high isolation yields and are not affected by the patients' age, sex and physiological status There have been many reports and attempts on the clinical application of HaMPCs in regenerative medicine perspective. In China and worldwide, there are also many important enterprises and research institutes, hospitals and governments concerned about the clinical application of HaMPCs. These will promote the clinical translation of HaMPCs related technologies reported in this paper. We suggest that in the future, larger scales of randomized clinical trials should be conducted to further verify the efficacy of haMPCs in human cartilage defects, and this technique should be extended to the repair of articular cartilage defects in shoulder, hip and ankle.

Supplementary data

To view the supplementary data that accompany this paper please visit the journal website at: www.futuremedicine.com/doi/sup $\mathrm{pl} / 10.2217 / \mathrm{rme}-2019-0068$

\section{Acknowledgments}

The authors express their sincere appreciation to the following individuals and institutions for the support and assistance in this research: M Xin from Radiology Department, Ren Ji Hospital; H Xiao from Radiology Department, Shanghai Ninth People's Hospital; 
Y Bao from Pathology Department, Shanghai Ninth People's Hospital. The authors also thank the patients who participated in this trial.

Financial \& competing interests disclosure

C Dai, S Li, M Li and C Xu are employees of Cellular Biomedicine Group Ltd. This study was funded by National Natural Science Foundation Youth Project of China (81701725), Joint research project on important diseases in Shanghai health system (2013ZYJB0501), SMC-Chenxing Young Scholar Award Scheme (AF4040017), and Shanghai municipal education commissionGaofeng clinical medicine grant support (20161423). The authors have no other relevant affiliations or financial involvement with any organization or entity with a financial interest in or financial conflict with the subject matter or materials discussed in the manuscript apart from those disclosed.

No writing assistance was utilized in the production of this manuscript.

\section{Ethical conduct of research}

This study was approved by the local ethics committees of both institutions (No.22, 2014; Scientific Research Projects Approval Determination of Independent Ethics Committee of Shanghai Ninth People's Hospital affiliated to Shanghai Jiao Tong University School of Medicine and Scientific Research Projects Approval Determination of Independent Ethics Committee of Renji Hospital Affiliated to Shanghai Jiao Tong University School of Medicine) and registered at clinicaltrials.gov with identifier NCT02855073. All procedures were conducted in accordance with the Declaration of Helsinki. The methods were carried out in accordance with the relevant guidelines and regulations. Written informed consent was obtained from all patients after the physicians illustrated detailed relevant information, such as the purpose and overall procedure of the study, potential risks and benefits.

\section{Data sharing statement}

The authors certify that this manuscript reports original clinical trial data. Data reported in this manuscript are available within the article or posted publicly at www.clinicaltrials.gov, according to the required timelines. Individual de-identified participant data which underlie results reported in this article will be available from 9 months and ending 24 months following article publication. The study protocol will also be available. Material will be accessible to investigators whose proposed use of data has been approved by an independent review committee identified for this purpose and for data meta-analysis. Requests are to be directed to the corresponding author of the article up to 24 months following article publication.

\section{Summary points}

- Intra-articular injections of human adipose-derived mesenchymal progenitor cell (haMPCs) correlated with a significant reduction of the Western Ontario and McMaster Universities Osteoarthritis index and improvement in the short form 36 score, when combined with microfracture surgery.

- Intra-articular injections of haMPCs in combination with hyaluronic acid and microfracture did not correlate with treatment-related serious adverse events.

- Intra-articular injections of haMPCs were associated with a significantly reduction of the articular cartilage defect and improvement in International Cartilage Repair Society grade under arthroscopy when combined with microfracture surgery.

- Intra-articular injections of haMPCs were associated with a significantly reduction of the articular cartilage defect area under magnetic resonance imaging and increased cartilage volume when combined with microfracture surgery.

- Intra-articular injections of haMPCs were safe and improved the clinical function, reduced cartilage defect and increased cartilage volume of the knee joint, encouraging larger scales of randomized clinical trials to further verify the efficacy of haMPCs, when combined with microfracture surgery.

\section{References}

1. Findlay DM. If good things come from above, do bad things come from below? Arthritis Res. Ther. 12(3), 119 (2010).

2. Wieland HA, Michaelis M, Kirschbaum BJ, Rudolphi KA. Osteoarthritis - an untreatable disease? Nat. Rev. Drug Discov. 4(4), 331-344 (2005).

3. Conaghan PG, Dickson J, Grant RL, . Care and management of osteoarthritis in adults: summary of NICE guidance. BMJ 336(7642), 502-503 (2008).

4. Zhang W, Nuki G, Moskowitz RW et al. OARSI recommendations for the management of hip and knee osteoarthritis: part III: changes in evidence following systematic cumulative update of research published through January 2009. Osteoarthr. Cartil. 18(4), 476-499 (2010). 
5. Sinusas K. Osteoarthritis: diagnosis and treatment. Am. Fam. Physician 85(1), 49-56 (2012).

6. Kreuz PC, Erggelet C, Steinwachs MR et al. Is microfracture of chondral defects in the knee associated with different results in patients aged 40 years or younger? Arthroscopy 22(11), 1180-1186 (2006).

7. Kreuz PC, Steinwachs MR, Erggelet $\mathrm{C}$ et al. Results after microfracture of full-thickness chondral defects in different compartments in the knee. Osteoarthr. Cartil. 14(11), 1119-1125 (2006).

8. Mithoefer K, Williams RJ, 3rd, Warren RF et al. The microfracture technique for the treatment of articular cartilage lesions in the knee. A prospective cohort study. J. Bone Joint Surg. Am. 87(9), 1911-1920 (2005).

9. Nguyen PD, Tran TD, Nguyen HT et al. Comparative clinical observation of arthroscopic microfracture in the presence and absence of a stromal vascular fraction injection for osteoarthritis. Stem Cells Translational Medicine 6(1), 187-195 (2017).

10. Steadman JR, Rodkey WG, Briggs KK. Microfracture to treat full-thickness chondral defects: surgical technique, rehabilitation, and outcomes. J. Knee Surg. 15(3), 170-176 (2002).

11. Arroll B, Goodyear-Smith F. Corticosteroid injections for osteoarthritis of the knee: meta-analysis. BMJ 328(7444), 869 (2004).

12. Neustadt DH. Long-term efficacy and safety of intra-articular sodium hyaluronate (Hyalgan) in patients with osteoarthritis of the knee. Clin. Exp. Rheumatol. 21(3), 307-311 (2003).

13. Waddell DD, Bricker DC. Clinical experience with the effectiveness and tolerability of hylan G-F 20 in 1047 patients with osteoarthritis of the knee. J. Knee Surg. 19(1), 19-27 (2006).

14. Hempfling H. Intra-articular hyaluronic acid after knee arthroscopy: a two-year study. Knee Surg. Sports Traumatol. Arthrosc. 15(5), 537-546 (2007).

15. Turajane T, Labpiboonpong V, Maungsiri S. Cost analysis of intra-articular sodium hyaluronate treatment in knee osteoarthritis patients who failed conservative treatment. J. Med. Assoc. Thai. 90(9), 1839-1844 (2007).

16. Phiphobmongkol V, Sudhasaneya V. The effectiveness and safety of intra-articular injection of sodium hyaluronate $(500-730 \mathrm{kDa})$ in the treatment of patients with painful knee osteoarthritis. J. Med. Assoc. Thai. 92(10), 1287-1294 (2009).

17. Miller LE, Block JE. US-approved intra-articular hyaluronic acid injections are safe and effective in patients with knee osteoarthritis: systematic review and meta-analysis of randomized, saline-controlled trials. Clin. Med. Insights Arthritis Musculoskelet. Disord. 6, 57-63 (2013).

18. Van Jonbergen HP, Poolman RW, Van Kampen A. Isolated patellofemoral osteoarthritis. Acta Orthop. 81(2), 199-205 (2010).

19. Healy WL, Della Valle CJ, Iorio R et al. Complications of total knee arthroplasty: standardized list and definitions of the Knee Society. Clin. Orthop. Relat. Res. 471(1), 215-220 (2013).

20. Rutjes AW, Juni P, Da Costa BR, Trelle S, Nuesch E, Reichenbach S. Viscosupplementation for osteoarthritis of the knee: a systematic review and meta-analysis. Ann. Intern. Med. 157(3), 180-191 (2012).

21. Koh YG, Choi YJ. Infrapatellar fat pad-derived mesenchymal stem cell therapy for knee osteoarthritis. Knee 19(6), 902-907 (2012).

22. Pak J, Chang JJ, Lee JH, Lee SH. Safety reporting on implantation of autologous adipose tissue-derived stem cells with platelet-rich plasma into human articular joints. BMC Musculoskelet. Disord. 14, 337 (2013).

23. Pak J, Lee JH, Lee SH. A novel biological approach to treat chondromalacia patellae. PLoS ONE 8(5), e64569 (2013).

24. Koh YG, Jo SB, Kwon OR et al. Mesenchymal stem cell injections improve symptoms of knee osteoarthritis. Arthroscopy 29(4), 748-755 (2013).

25. Pak J. Regeneration of human bones in hip osteonecrosis and human cartilage in knee osteoarthritis with autologous adipose-tissue-derived stem cells: a case series. J. Med. Case Rep. 5, 296 (2011).

26. Koh YG, Choi YJ, Kwon SK, Kim YS, Yeo JE. Clinical results and second-look arthroscopic findings after treatment with adipose-derived stem cells for knee osteoarthritis. Knee Surg. Sports Traumatol. Arthrosc. 23(5), 1308-1316 (2015).

27. Kirkley A, Birmingham TB, Litchfield RB et al. A randomized trial of arthroscopic surgery for osteoarthritis of the knee. N. Engl. J. Med. 359(11), 1097-1107 (2008).

28. Risberg MA. Arthroscopic surgery provides no additional benefit over physiotherapy and medication for the treatment of knee osteoarthritis. Aust. J. Physiother. 55(2), 137 (2009).

29. Jevsevar D, Donnelly P, Brown GA, Cummins DS. Viscosupplementation for osteoarthritis of the knee: a systematic review of the evidence. J. Bone Joint Surg. Am. 97(24), 2047-2060 (2015).

30. Healy WL, Iorio R, Lemos MJ. Athletic activity after joint replacement. Am. J. Sports Med. 29(3), 377-388 (2001).

31. Papalia R, Del Buono A, Zampogna B, Maffulli N, Denaro V. Sport activity following joint arthroplasty: a systematic review. Br. Med. Bull. 101, 81-103 (2012).

32. Bradbury N, Borton D, Spoo G, Cross MJ. Participation in sports after total knee replacement. Am. J. Sports Med. 26(4), 530-535 (1998).

33. Steadman JR, Briggs KK, Matheny LM, Ellis HB. Ten-year survivorship after knee arthroscopy in patients with Kellgren-Lawrence grade 3 and grade 4 osteoarthritis of the knee. Arthroscopy 29(2), 220-225 (2013). 
34. Filardo G, Madry H, Jelic M, Roffi A, Cucchiarini M, Kon E. Mesenchymal stem cells for the treatment of cartilage lesions: from preclinical findings to clinical application in orthopaedics. Knee Surg. Sports Traumatol. Arthrosc. 21(8), 1717-1729 (2013).

35. Jorgensen C, Djouad F, Bouffi C, Mrugala D, Noel D. Multipotent mesenchymal stromal cells in articular diseases. Best Pract. Res. Clin. Rheumatol. 22(2), 269-284 (2008).

36. Lopez-Ruiz E, Peran M, Cobo-Molinos J et al. Chondrocytes extract from patients with osteoarthritis induces chondrogenesis in infrapatellar fat pad-derived stem cells. Osteoarthr. Cartil. 21(1), 246-258 (2013).

37. Faustini M, Bucco M, Chlapanidas $\mathrm{T}$ et al. Nonexpanded mesenchymal stem cells for regenerative medicine: yield in stromal vascular fraction from adipose tissues. Tissue Eng Part C Methods 16(6), 1515-1521 (2010).

38. Wang W, He N, Feng $\mathrm{C}$ et al. Human adipose-derived mesenchymal progenitor cells engraft into rabbit articular cartilage. Int. J. Mol. Sci. 16(6), 12076-12091 (2015).

39. Xu X, Shi D, Liu Y et al. In vivo repair of full-thickness cartilage defect with human iPSC-derived mesenchymal progenitor cells in a rabbit model. Exp. Ther. Med. 14(1), 239-245 (2017).

40. Kellgren JH, Lawrence JS. Radiological assessment of osteo-arthrosis. Ann. Rheum. Dis. 16(4), 494-502 (1957).

41. Mao ZH, Yin JH, Zhang XX, Wang X, Xia Y. Discrimination of healthy and osteoarthritic articular cartilage by Fourier transform infrared imaging and Fisher's discriminant analysis. Biomed. Opt. Express 7(2), 448-453 (2016).

42. Van Den Borne MP, Raijmakers NJ, Vanlauwe J et al. International Cartilage Repair Society (ICRS) and Oswestry macroscopic cartilage evaluation scores validated for use in Autologous Chondrocyte Implantation (ACI) and microfracture. Osteoarthr. Cartil. 15(12), 1397-1402 (2007).

43. Bellamy N, Buchanan WW, Goldsmith CH, Campbell J, Stitt LW. Validation study of WOMAC: a health status instrument for measuring clinically important patient relevant outcomes to antirheumatic drug therapy in patients with osteoarthritis of the hip or knee. J. Rheumatol. 15(12), 1833-1840 (1988).

44. Ware JE, Jr, Sherbourne CD. The MOS 36-item short-form health survey (SF-36). I. Conceptual framework and item selection. Med. Care 30(6), 473-483 (1992).

45. Jo CH, Lee YG, Shin WH et al. Intra-articular injection of mesenchymal stem cells for the treatment of osteoarthritis of the knee: a proof-of-concept clinical trial. Stem Cells 32(5), 1254-1266 (2014).

46. Brittberg M, Peterson L. Introduction of an articular cartilage classification. ICRS Newsletter 1(5-8), (1998).

47. Peterson L, Minas T, Brittberg M, Nilsson A, Sjogren-Jansson E, Lindahl A. Two- to 9-year outcome after autologous chondrocyte transplantation of the knee. Clin. Orthop. Relat. Res. doi:10.1097/00003086-200005000-00020(374), 212-234 (2000).

48. Mainil-Varlet P, Van Damme B, Nesic D, Knutsen G, Kandel R, Roberts S. A new histology scoring system for the assessment of the quality of human cartilage repair: ICRS II. Am. J. Sports Med. 38(5), 880-890 (2010).

49. Giri S, Santosha, Singh CHA et al. Role of arthroscopy in the treatment of osteoarthritis of knee. J. Clin. Diagn. Res. JCDR 9(8), RC08-11 (2015).

50. Thorlund JB, Juhl CB, Roos EM, Lohmander LS. Arthroscopic surgery for degenerative knee: systematic review and meta-analysis of benefits and harms. Br. J. Sports Med. 49(19), 1229-1235 (2015).

51. Frisbie DD, Oxford JT, Southwood L et al. Early events in cartilage repair after subchondral bone microfracture. Clin. Orthop. Relat. Res. doi:10.1097/00003086-200302000-00031(407), 215-227 (2003).

52. Frisbie DD, Trotter GW, Powers BE et al. Arthroscopic subchondral bone plate microfracture technique augments healing of large chondral defects in the radial carpal bone and medial femoral condyle of horses. Vet. Surg. 28(4), 242-255 (1999).

53. Gobbi A, Nunag P, Malinowski K. Treatment of full thickness chondral lesions of the knee with microfracture in a group of athletes. Knee Surg. Sports Traumatol. Arthrosc. 13(3), 213-221 (2005).

54. Knutsen G, Engebretsen L, Ludvigsen TC et al. Autologous chondrocyte implantation compared with microfracture in the knee. A randomized trial. J. Bone Joint Surg. Am. 86(3), 455-464 (2004).

55. Steadman JR, Briggs KK, Rodrigo JJ, Kocher MS, Gill TJ, Rodkey WG. Outcomes of microfracture for traumatic chondral defects of the knee: average 11-year follow-up. Arthroscopy 19(5), 477-484 (2003).

56. Steadman JR, Miller BS, Karas SG, Schlegel TF, Briggs KK, Hawkins RJ. The microfracture technique in the treatment of full-thickness chondral lesions of the knee in National Football League players. J. Knee Surg. 16(2), 83-86 (2003).

57. Steadman JR, Rodkey WG, Rodrigo JJ. Microfracture: surgical technique and rehabilitation to treat chondral defects. Clin. Orthop. Relat. Res. doi:10.1097/00003086-200110001-00033 (391 Suppl.), S362-S369 (2001).

58. Steadman JR, Rodkey WG, Singleton SB, Briggs KK. Microfracture technique forfull-thickness chondral defects: technique and clinical results. Oper. Tech. Orthop. 7(4), 300-304 (1997).

59. Pers YM, Rackwitz L, Ferreira R et al. Adipose mesenchymal stromal cell-based therapy for severe osteoarthritis of the knee: a Phase I dose-escalation trial. Stem cells translational medicine 5(7), 847-856 (2016).

60. Berenbaum F, Grifka J, Cazzaniga $S$ et al. A randomised, double-blind, controlled trial comparing two intra-articular hyaluronic acid preparations differing by their molecular weight in symptomatic knee osteoarthritis. Ann. Rheum. Dis. 71(9), 1454-1460 (2012). 
61. Zhang H, Zhang K, Zhang X et al. Comparison of two hyaluronic acid formulations for safety and efficacy (CHASE) study in knee osteoarthritis: a multicenter, randomized, double-blind, 26-week non-inferiority trial comparing Durolane to Artz. Arthritis Res. Ther. 17, 51 (2015). 